\title{
Remarks on heavy-light mesons from AdS/CFT
}

\author{
Christopher P. Herzog, ${ }^{a}$ Stefan A. Stricker ${ }^{b}$ and Aleksi Vuorinen ${ }^{b c}$ \\ a Joseph Henry Laboratories, Princeton University, \\ Washington Road, Princeton, NJ 08544, U.S.A. \\ ${ }^{b}$ Institut für Theoretische Physik, Technische Universität Wien, \\ Wiedner Hauptstr. 8-10, A-1040 Vienna, Austria \\ ${ }^{c}$ CERN, Physics Department, TH Unit, \\ CH-1211 Geneva 23, Switzerland \\ E-mail: cpherzog@princeton.edu, stricker@hep.itp.tuwien.ac.at, \\ vuorinen@phys.washington.edu
}

ABSTRACT: We use the AdS/CFT correspondence to compute the energy spectrum of heavy-light mesons in a $\mathcal{N}=2 \mathrm{SU}(N)$ super Yang-Mills theory with two massive hypermultiplets. In the heavy quark limit, similar to QCD, we find that the excitation energies are independent of the heavy quark mass. We also make some remarks about related AdS/CFT models of flavor with less supersymmetry.

KEYwords: AdS-CFT Correspondence, Heavy Quark Physics. 


\section{Contents}

1. Introduction 1

2. Supersymmetry considerations 1

3. Mass spectra of heavy-light mesons: preliminaries 7

4. Fluctuations in $x, \rho$ and $y_{6}$

4.1 The $x$ fluctuations 10

4.2 The $y_{6}$ fluctuations 11

4.3 The $\delta \rho$ fluctuations 11

4.4 The meson mass spectrum

5. Spinning strings

5.1 Strings spinning in real space 16

5.2 String profile in $\rho$ and $\theta$

6. Summary and discussion 26

\section{Introduction}

The heavy quark limit of QCD has been an important tool in understanding the spectrum and decays of mesons and baryons with a heavy quark constituent; see ref. [1] for a review. When the mass of the heavy quark is large compared to the QCD scale, $m_{h} \gg \Lambda_{\mathrm{QCD}}$, the interaction between the heavy quark and the light quarks and gluons becomes independent of the spin and flavor of the heavy quark. This independence yields predictions for the $m_{h}$ dependence of the meson spectrum and weak decay amplitudes. In this paper, we investigate the heavy quark limit not in QCD but in a cousin of $\mathcal{N}=4 \mathrm{SU}(N)$ super Yang-Mills (SYM) theory. We add two fundamental hypermultiplets, with masses $m_{l}$ and $m_{h}$, to $\mathcal{N}=4 \mathrm{SYM}$, breaking the supersymmetry to $\mathcal{N}=2$. Using the AdS/CFT correspondence [3-5], we study the spectrum of heavy-light mesons in this theory at large $N$ and large 't Hooft coupling $\lambda=g_{\mathrm{YM}}^{2} N$.

One reason why heavy quarks are easier to understand in QCD than light quarks is asymptotic freedom; at short distance scales and high energies, the strong force becomes weak. Roughly speaking, for energies sufficiently above $\Lambda_{\mathrm{QCD}}$, the coupling constant $\alpha_{s}$ becomes small, and thus the interactions of the heavy quarks, charm, bottom and top, are governed by a weak effective coupling $\alpha_{s}\left(m_{h}\right)$. The light quarks, up, down, and strange, on the other hand experience a much stronger coupling $\alpha_{s}(\Lambda)$, with $\Lambda$ only slightly above 
$\Lambda_{\mathrm{QCD}}$, where the coupling diverges. Indeed, the strong force between two heavy quarks is weak enough to be treated perturbatively, and is similar to the force between an electron and a positron. Heavy-heavy mesons, which are bound states of two heavy quarks, therefore have measured properties very similar to positronium. ${ }^{1}$

Heavy-light mesons, in contrast, are more complicated objects, as their light quark constituent experiences strong interactions. Qualitatively, the heavy quark is a small object of size $1 / m_{h}$ surrounded by a "brown muck" of size $1 / \Lambda_{\mathrm{QCD}}$ of virtual strongly interacting light quarks, antiquarks, and gluons. However, the small size of the heavy quark leads to simplifications. The "brown muck" cannot resolve the spin or flavor of the heavy quark to leading order in $1 / m_{h}$, which means the interaction is spin and flavor blind.

The current paper was motivated by wondering, what parallels exist between heavylight mesons in real world QCD and in strongly coupled $\mathcal{N}=2 \mathrm{SU}(N)$ SYM theory with two massive hypermultiplets. The parent theory $\mathcal{N}=4 \mathrm{SU}(N)$ SYM is clearly very different from QCD. Most importantly for our comparison, $\mathcal{N}=4 \mathrm{SYM}$ is conformal, and we thus have no equivalent notion of the coupling constant being $m_{h}$ dependent. We also have no notion of a confinement or QCD scale $\Lambda_{\mathrm{QCD}}$; for us the IR scale will be $m_{l}$. It is true that adding $N_{f}=2$ hypermultiplets to $\mathcal{N}=4$ SYM breaks the conformal symmetry, but the nonzero beta function in fact runs in the wrong direction, toward strong coupling in the UV. In this paper, we will, however, work in the limit $N_{f} \ll N$, and therefore ignore $N_{f} / N$ suppressed effects.

Despite these differences, there is persistent hope that we may gain insights into QCD by asking the right questions about $\mathcal{N}=4 \mathrm{SYM}$ and its relatives at strong coupling. For example, at zero temperature, the Klebanov-Strassler model [6] provides a geometric understanding of abelian chiral symmetry breaking and confinement for a $\mathcal{N}=1$ supersymmetric gauge theory in this AdS/CFT context. Regarding nonzero temperature physics, where the arguments are perhaps more compelling, ref. [7] made the following two observations. First, consider the ratio of the pressure at strong and weak coupling. The ratio for $\mathcal{N}=4$ SYM was computed by ref. [8] to be $3 / 4$. QCD is not conformal, but lattice simulations can be used to compute the pressure at a few times the deconfinement temperature where the theory is relatively strongly interacting and the pressure slowly varying. The ratio of this pressure to the free result is about 0.8 . The second observation is that at strong coupling, both $\mathcal{N}=4$ SYM and QCD are believed to have very small viscosities (see e.g. refs. [10, 9]).

The AdS/CFT correspondence maps $\mathcal{N}=4 \mathrm{SU}(N)$ SYM theory to type IIB string theory in the curved background $A d S_{5} \times S^{5}$. We will work in the large $N$ and $\lambda$ limit, where the string theory becomes classical and can be well approximated by supergravity. As described by ref. [2], a hypermultiplet can be added to the gauge theory by placing a D7 brane in the dual geometry. The heavy-light mesons we consider then, according to the duality, correspond to strings stretching between two parallel D7 branes, and the energy spectrum consists of the vibrational and rotational modes of the strings. Consistent with

\footnotetext{
${ }^{1}$ Note however that highly excited charmonium and bottomonium states are expected to be sensitive to the details of confinement. For these excited states, the quarks are separated by relatively large distances and experience a linear confining potential rather than a Coulombic potential. To reproduce the full spectrum, the Cornell potential, which interpolates between these two limiting forms, is often used.
} 
our large $N$ limit, we will neglect the back reaction of the $\mathrm{D}$ branes on the geometry, as well as the back reaction of the strings on the D branes and the geometry.

Despite the conformal nature of the theory we consider, we find that the meson spectrum is, in an appropriate sense, spin and flavor blind in the heavy quark and strong coupling limit. The mass $M_{\mathrm{hl}}$ of the heavy-light mesons we find has the form

$$
M_{h l}=m_{h}+m_{l} f\left(\frac{J}{\sqrt{\lambda}}, \frac{Q}{\sqrt{\lambda}}, \frac{n}{\sqrt{\lambda}}\right)+\mathcal{O}\left(\frac{m_{l}^{2}}{m_{h}}\right),
$$

where $J$ is the angular momentum of the meson, $Q$ an R-charge, and $n$ a quantum number specifying a radial excitation. ${ }^{2}$ We have not introduced a confinement scale and thus $m_{l}$ takes the place of $\Lambda_{\mathrm{QCD}}$.

One important aspect of this heavy-light meson spectrum is its $m_{h}$ independence, which can be understood in the following way. The excitations (at least in $n$ and $J$ ) we find are closely analogous to the modes of a guitar string, the length of which is proportional to $1 / m_{l}-1 / m_{h}$. In the heavy quark limit, the length of the string becomes independent of $1 / m_{h}$, and hence it is expected that also the frequencies of the modes become $1 / m_{h}$ independent.

After the appearance of ref. [2], there have been many detailed studies of the meson spectrum of the $\mathcal{N}=2 \mathrm{SU}(N) \mathrm{SYM}$ theory beginning with refs. [11, 12]. In fact, a nice review 13 has appeared to which we point the interested reader for a more complete list of references. To understand what is new about the current paper, it is useful to outline the differences of our work from ref. [12], where the authors considered the meson spectrum for $\mathcal{N}=2 \mathrm{SYM}$ theory with a single massive hypermultiplet of mass $m$. They considered two different types of mesons. The first type have a very small mass $M \sim m / \sqrt{\lambda}$ and spin $0,1 / 2$, or 1 , and are dual to fluctuations of the D7 brane embedding. The second type are dual to U-shaped semiclassical strings with much larger angular momentum $J$ and mass. For $J \ll \sqrt{\lambda}$, the mass obeys Regge scaling $M \sim m \sqrt{J} / \lambda^{1 / 4}$ while for $J \gg \sqrt{\lambda}$, the potential is Coulombic $M=2 m$ - const $/ J^{2}$. While the behavior of these types of mesons are qualitatively diffferent, there is expected to be a way in which as we consider mesons with larger and larger angular momentum, the D7 brane fluctuations in fact morph into semiclassical string configurations.

The ground state of our heavy-light meson is a string, which stretches between two D7 branes separated by a finite distance proportional to the mass difference between the hypermultiplets. Having taken the heavy-quark limit, there is no sense in which our meson spectrum is well approximated by D7 brane fluctuations. To find the spectrum, we therefore instead consider fluctuations of the string itself, which will correspond to radial excitations of the meson. We also consider the dependence of the string energy on its angular momentum $J$ and charge $Q$, and this part of the analysis is similar to the second half of ref. [12] and section 2 of [14].

The types of heavy-light mesons we consider have been studied before, in refs. [14[16]. Ref. [14], is very similar in spirit to ours. Indeed, section 2 of ref. [14] overlaps to

\footnotetext{
${ }^{2}$ Recall that $\mathcal{N}=2$ supersymmetric gauge theories have a global R-symmetry. Geometrically, $Q$ is an angular momentum in the internal $S^{5}$.
} 
some extent with our discussion of the spinning strings in section 5.1. In refs. [15, 16], it was pointed out that the ground state heavy-light mesons have a mass which scales as the difference of the heavy quark masses, $M=m_{h}-m_{l}$. This scaling is very different from the D7 brane fluctuations considered in ref. [12], which yielded masses $M \sim m / \sqrt{\lambda}$ for the heavy-heavy and light-light mesons. Ref. [15] also demonstrated that the excitation energies above the ground state are suppressed by a power of $\lambda$. This work should in principle be very similar to what we do here, as the authors of ref. [15] also study the fluctuation spectrum of a semiclassical string stretching between two D7 branes in the $A d S_{5} \times S^{5}$ geometry. However, they work in an approximation where the strings do not bend and find that the excitation energies for heavy-light mesons scale with $m_{h}$ instead of $m_{l}$. Ref. [16] in contrast is a calculation in a different limit: They consider the case where the masses of the two hypermultiplets become degenerate and thus non-abelian effects on the D7 branes are important.

Our paper is organized as follows. We begin in section 2 by reviewing the dual supergravity construction of $\mathcal{N}=2 \mathrm{SYM}$ theory with two massive fundamental hypermultiplets, and in addition we make some remarks about related constructions that preserve only $\mathcal{N}=1$ supersymmetry, allowing for a novel way of thinking about meson decay and also yielding a spectrum of heavy-light mesons similar to the spectrum of the heavy-heavy and light-light mesons considered in [12]. In the following sections we consider only the $\mathcal{N}=2$ supersymmetry preserving case. Section 3 fixes our notation and sets up the supergravity calculation of the heavy-light meson spectrum.

In section 4, we analyze small fluctuations of the string dual to the heavy-light meson and thus obtain the spectrum as a function of what we called $n$ above. This analysis ignores nonlinearities in the equation of motion for the string and is valid when the occupation numbers of the modes are small compared to $\sqrt{\lambda}$. Section 5 follows with a discussion of spinning strings dual to heavy-light mesons with large charge and angular momentum. The analysis is purely classical but employs the full nonlinear equations of motion. We expect a classical analysis to be valid in the limit where $J \gg 1$ and $Q \gg 1$, but we also find that the solutions match smoothly onto the small fluctuations considered in section 1 at small values of $J$ and $Q$. The paper concludes with a comparison to the spectrum of real world (QCD) heavy-light mesons in the summary section.

\section{Supersymmetry considerations}

We know that type IIB strings in an $A d S_{5} \times S^{5}$ space-time are dual to $\mathcal{N}=4 \mathrm{SU}(N)$ super Yang-Mills theory through the AdS/CFT correspondence. The space $A d S_{5} \times S^{5}$ has the line element

$$
d s^{2}=L^{2}\left[u^{2} \eta_{\mu \nu} d x^{\mu} d x^{\nu}+\frac{\delta_{i j} d y^{i} d y^{j}}{u^{2}}\right],
$$

where the indices $i$ and $j$ run from one to six, $\mu$ and $\nu$ run from zero to three, and $L$ is the radius of curvature. The coordinate $u^{2} \equiv \sum_{i}\left(y^{i}\right)^{2}$ is a radial coordinate, and as $u \rightarrow \infty$, we reach the boundary of $A d S_{5}$. In this notation, the metric is clearly a warped product of Minkowski space $\mathbb{R}^{1,3}$ with $\mathbb{R}^{6}$. The line element can also be written to make the $A d S_{5}$ 
more explicit:

$$
d s^{2}=\frac{L^{2}}{z^{2}}\left(\eta_{\mu \nu} d x^{\mu} d x^{\nu}+d z^{2}\right)+L^{2} d \Omega^{2},
$$

where $d \Omega^{2}$ is a line element on the $S^{5}$ and $u=1 / z$. The $\mathrm{SO}(6)$ isometry group of the $S^{5}$ geometrically realizes the $\mathrm{SO}(6) \mathrm{R}$-symmetry of the dual field theory.

As described by Karch and Katz [2], adding an $\mathcal{N}=2$ hypermultiplet to the gauge theory is dual to placing a D7 brane in the dual geometry. The D7 brane spans the Minkowski directions $x^{\mu}$ and four of the remaining directions in $\mathbb{R}^{6}$. With this ansatz, the D7 brane is insensitive to the RR-five form flux in the curved geometry, and its behavior is determined solely through the DBI action

$$
S_{\mathrm{DBI}}=-\tau_{7} \int d^{8} \xi \sqrt{-\operatorname{det}\left(G_{a b}+2 \pi \alpha^{\prime} \mathcal{F}_{a b}\right)},
$$

where $\tau_{7}=1 /(2 \pi)^{7} \alpha^{\prime 4} g_{s}$ is the D7 brane tension, $1 / 2 \pi \alpha^{\prime}$ is the string tension, $g_{s}$ is the string coupling constant, $G_{a b}$ is the induced metric on the D7 brane, and $\mathcal{F}_{a b}$ is the gauge field on the D7 brane. We will consider only the case $\mathcal{F}_{a b}=0$ in these remarks. Recall that the AdS/CFT dictionary relates

$$
\frac{L^{2}}{\alpha^{\prime}}=\sqrt{\lambda} \text { and } 4 \pi g_{s}=g_{\mathrm{YM}}^{2},
$$

where $\lambda=g_{\mathrm{YM}}^{2} N$ is the 't Hooft coupling.

To correspond to a hypermultiplet, the D7 brane must span $\mathbb{R}^{1,3}$, and thus the four remaining dimensions of the $\mathrm{D} 7$ brane lie in $\mathbb{R}^{6}$. It seems natural to choose a gauge in which four of the coordinates on the D7 brane are the $x^{\mu}$. Moreover we pick an embedding in $\mathbb{R}^{6}$ that does not depend on the $x^{\mu}$. Given this independence, the determinant of the induced metric on the D7 brane will not depend on the warp factor $u^{2}$ in the ten dimensional metric (2.1). Dividing out by the volume of Minkowski space, the DBI action can be written in the form

$$
S_{\mathrm{DBI}}=-\tau_{7} L^{8} \int d^{4} \xi \sqrt{\operatorname{det}\left(\frac{\partial \mathbf{y}}{\partial \xi^{a}} \cdot \frac{\partial \mathbf{y}}{\partial \xi^{b}}\right)} .
$$

The D7 brane will satisfy the same equations of motion that it does in flat space; the D7 brane describes a minimal four dimensional hypersurface in $\mathbb{R}^{6}$. Note that the normalization of the DBI action can be written in gauge theory language as

$$
\tau_{7} L^{8}=\frac{2 \lambda N}{(2 \pi)^{6}} .
$$

The DBI action is smaller by a factor of $N$ compared to the supergravity action, justifying our neglect of the back reaction of the D7 brane on the geometry.

A particularly simple class of hypersurfaces which satisfy the equations of motion are surfaces described by a holomorphic embedding equation. If we think of $\mathbb{R}^{6}=\mathbb{C}^{3}$ as a complex manifold and define coordinates $w^{j}=y^{2 j-1}+i y^{2 j}$, a D7 brane which satisfies an equation of the form $f\left(w^{1}, w^{2}, w^{3}\right)=0$ for an arbitrary function $f$ will locally satisfy the equations of motion away from singularities. 
The Karch-Katz D7 brane is a hyperplane described by two linear equations $\mathbf{a}_{1} \cdot \mathbf{y}=c_{1}$ and $\mathbf{a}_{2} \cdot \mathbf{y}=c_{2}$. Given the $\mathrm{SO}(6)$ rotational symmetry of the sphere, such a hyperplane can be rotated so that the two equations become $y^{5}=c$ and $y^{6}=0 .{ }^{3}$ In complex coordinates, the hyperplane is the complex submanifold described by $f=w^{3}-c$. The parameter $c$ is dual to the mass of the hypermultiplet.

The Karch-Katz D7 brane preserves $\mathcal{N}=2$ supersymmetry, while the more general case $f\left(w^{1}, w^{2}, w^{3}\right)=0$ preserves only $\mathcal{N}=1$ supersymmetry (see e.g. ref. [21]). In brief, there are 32 real spinors generating supersymmetry transformations that leave invariant the $A d S_{5} \times S^{5}$ type IIB supergravity background, 16 of which correspond to ordinary supercharges and the remainder of which are superconformal. This number of supercharges is sufficient to generate the $\mathcal{N}=4$ superconformal algebra of the dual Yang-Mills field theory. Of these 32 spinors, only four of the ordinary and none of the superconformal generate supersymmetry transformations which leave a general D7 brane satisfying $f\left(w^{1}, w^{2}, w^{3}\right)=0$ invariant. The four invariant spinors are independent of the choice of $f\left(w^{1}, w^{2}, w^{3}\right)$. The Karch-Katz D7 brane, on the other hand, is left invariant by 8 of the ordinary spinors.

Given that a single Karch-Katz D7 brane corresponds to adding a single $\mathcal{N}=2$ hypermultiplet, adding two such D7 branes should correspond to adding two hypermultiplets. In the literature [15- 17], we find that the second D7 brane is usually added in a way such that the embedding equation for the second D7 brane is parallel to the first, $w^{3}=c^{\prime}$ where $c^{\prime} \in \mathbb{R}$. Adding the second D7 brane in such a way has a number of desirable features. The theory remains $\mathcal{N}=2$ supersymmetric. Moreover, an unbroken $\mathrm{SO}(4) \subset \mathrm{SO}(6)$ of the global R-symmetry is preserved. Note that $c^{\prime} \in \mathbb{C}$ still preserves $\mathcal{N}=2$ supersymmetry and the $\mathrm{SO}(4) \mathrm{R}$-symmetry. The relative phase of $c$ and $c^{\prime}$ affects the relative phase of the hypermultiplet masses and also the mass of the heavy-light meson, a fact we will return to in the discussion.

However, a generic second D7 brane would not be parallel to the first. Assuming the second D7 brane is also described by a four dimensional hyperplane inside $\mathbb{R}^{6}$, the two D7 branes will generically intersect along a plane $\mathbb{R}^{2}$. Such an intersection generically breaks all the supersymmetry. If supersymmetry is broken, then there will probably be a tachyon, i.e. an instability, and the D7 branes will recombine; it's not clear what the final state will be, and we have little to say about this nonsupersymmetric situation.

While the remaining $\mathrm{SO}(4)$ symmetry is not enough to guarantee the second KarchKatz D7 brane can be described by a complex equation as well, there will be a special case where both D7 brane embeddings are described by complex equations in $\mathbb{C}^{3}$. This special case preserves $\mathcal{N}=1$ supersymmetry. Indeed, if we add any number of KarchKatz D7 branes such that they are all described by complex equations in $\mathbb{C}^{3}, \mathcal{N}=1$ supersymmetry is preserved. The reason is that the four spinors preserved by both the supergravity background and the D7 brane are independent of the choice of $f\left(w^{1}, w^{2}, w^{3}\right)$. These intersecting brane configurations should lead to a heavy-light meson spectrum similar to the heavy-heavy and light-light meson spectra found in ref. 12]. There will be short

\footnotetext{
${ }^{3}$ Use the $\mathrm{SO}(6)$ symmetry to rotate $\mathbf{a}_{1}$ into the $y^{5}$ direction and $\mathbf{a}_{2}$ into the $y^{5}-y^{6}$ plane. The problem reduces to considering the intersection of two lines in a plane. There is a residual $\mathrm{SO}(2)$ symmetry in the $y^{5}-y^{6}$ plane which always allows us to rotate the intersection point onto the $y^{5}$ axis.
} 
strings localized at the intersection of the two D branes whose masses should scale as the distance of the intersection from the origin of the geometry divided by $\sqrt{\lambda}$. These intersecting configurations also provide a novel way of thinking about meson decay, which is different from what has been considered in the literature before 18, 19. The case of three intersecting Karch-Katz D7 branes would be especially interesting to consider because the intersection of three four dimensional hyperplanes in $\mathbb{R}^{6}$ is in general a point. We, however, leave a study of such spectra and decays for the future.

Finally, we make a short remark on the field theory aspects of the system we are studying. We know that $\mathcal{N}=4 \mathrm{SU}(N)$ SYM has the superpotential

$$
W=\operatorname{Tr} X[Y, Z]
$$

where $X, Y$, and $Z$ are chiral superfields transforming in the adjoint of $\mathrm{SU}(N)$. The Karch-Katz D7 brane leads to the modified superpotential

$$
W=\operatorname{Tr} X[Y, Z]+\tilde{Q}(m-X) Q,
$$

where $Q$ and $\tilde{Q}$ are chiral superfields that transform in the fundamental of $\operatorname{SU}(N)$ and combine to form a hypermultiplet. ${ }^{4}$ The $\mathcal{N}=2$ supersymmetry preserving case of two parallel D7 branes has the superpotential

$$
W=\operatorname{Tr} X[Y, Z]+\tilde{Q}_{h}\left(m_{h}-X\right) Q_{h}+\tilde{Q}_{l}\left(m_{l}-X\right) Q_{l} .
$$

When $m_{h}$ and $m_{l}$ are both real, we chose above both $c$ and $c^{\prime} \in \mathbb{R}$. However, we may introduce a relative phase between $m_{h}$ and $m_{l}$ as well corresponding to $c^{\prime} \in \mathbb{C}$. Adding the D7 branes in a way that preserves only $\mathcal{N}=1$ superysmmetry corresponds to more general types of superpotentials, for example

$$
W=\operatorname{Tr} X[Y, Z]+\tilde{Q}_{h}\left(m_{h}-X\right) Q_{h}+\tilde{Q}_{l}\left(m_{l}-Y\right) Q_{l} .
$$

In most of the rest of what follows, we will restrict to the case where $m_{h}$ and $m_{l}$ are real and the two D7 branes preserve $\mathcal{N}=2$ supersymmetry.

\section{Mass spectra of heavy-light mesons: preliminaries}

We consider the special configuration of two parallel D7 branes in the $\mathcal{N}=2$ supersymmetric scenario described above where the ground state string will have a nonzero length. The string hangs from one brane to the other and the string endpoints correspond to one heavy and one light quark. Our aim is to derive the mass spectrum of heavy-light mesons by investigating the spectrum of fluctuations of strings hanging between the branes.

The $A d S_{5} \times S_{5}$ metric (2.1) can be thought of as a warped product metric on $\mathbb{R}^{1,3} \times \mathbb{R}^{6}$. We will write the line element on $\mathbb{R}^{6}$ as

$$
\delta_{i j} d y^{i} d y^{j}=d \rho^{2}+\rho^{2} d \theta^{2}+\rho^{2} \sin ^{2} \theta d \Omega_{2}^{2}+d y^{2}+\left(d y^{6}\right)^{2},
$$

\footnotetext{
${ }^{4}$ We have been careless of the relative normalizations of the different terms in $W$, but they will be fixed by supersymmetry. See e.g. ref. [20] for details.
} 


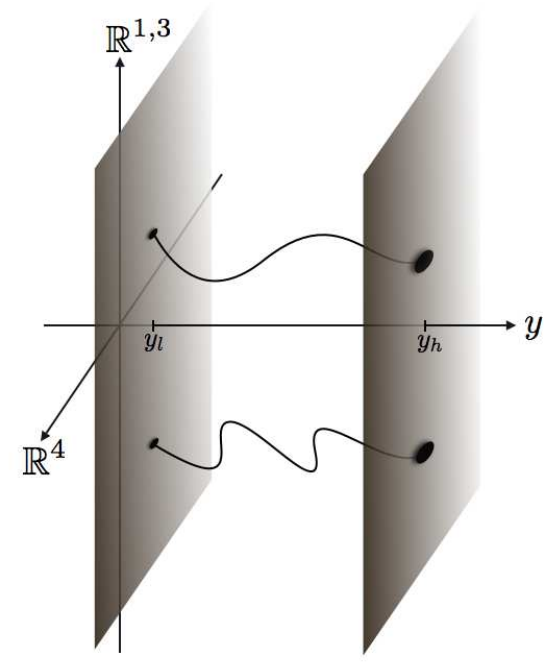

Figure 1: A cartoon of our heavy-light mesons as strings stretched between two D7 branes.

where $d \Omega_{2}^{2}$ is a metric on a unit $S^{2}$ and we have defined $\rho^{2} \equiv u^{2}-\left(y^{5}\right)^{2}-\left(y^{6}\right)^{2}$ and $y \equiv y^{5}$. The metric on Minkowski space $\mathbb{R}^{1,3}$ we will write as

$$
\eta_{\mu \nu} d x^{\mu} d x^{\nu}=-d t^{2}+d r^{2}+r^{2} d \phi^{2}+d x^{2} .
$$

In this geometry, strings that stretch from one D7 brane to another are dual to mesons, as illustrated in figure 1 which displays our geometric picture of heavy-light mesons. Classical strings are described by the Nambu-Goto action

$$
S_{\mathrm{NG}}=\int d \tau d \sigma \mathcal{L}=-\frac{1}{2 \pi \alpha^{\prime}} \int d \tau d \sigma \sqrt{\left(\dot{X} \cdot X^{\prime}\right)^{2}-(\dot{X})^{2}\left(X^{\prime}\right)^{2}},
$$

where $X^{A}(\tau, \sigma)$ describes the embedding of the string in $A d S_{5} \times S^{5}$. In our notation, $X \cdot Y=g_{A B} X^{A} Y^{B}$ is contracted with the ten dimensional metric, and we have defined $\partial_{\sigma} X \equiv X^{\prime}$ and $\partial_{\tau} X \equiv \dot{X}$. We choose a gauge in which the worldsheet coordinates are $\tau=t, \sigma=y$. The locations of the light and heavy D7 branes will be denoted by $y=y_{l}$ and $y=y_{h}$, and the light and heavy quark masses [2] read

$$
m_{l}=\frac{L^{2}}{2 \pi \alpha^{\prime}} y_{l} ; \quad m_{h}=\frac{L^{2}}{2 \pi \alpha^{\prime}} y_{h}
$$

where $L^{2} / \alpha^{\prime}=\sqrt{\lambda}$. The Nambu-Goto action is suppressed by a relative power of $N$ with respect to the DBI action, and thus we are justified in neglecting the back reaction of the string on the D7 brane and the geometry in the large $N$ limit.

We wish to study the profile that a string stretching between the D7 branes takes, assuming that the string sits at a constant position in the internal unit $S^{2}$. The Nambu- 
Goto action (3.3) produces the equation of motion

$$
0=\frac{\partial}{\partial \tau}\left(g_{A B} \frac{\left(\dot{X} \cdot X^{\prime}\right)\left(X^{\prime}\right)^{B}-\left(X^{\prime}\right)^{2} \dot{X}^{B}}{\sqrt{\left(\dot{X} \cdot X^{\prime}\right)^{2}-(\dot{X})^{2}\left(X^{\prime}\right)^{2}}}\right)+\frac{\partial}{\partial \sigma}\left(g_{A B} \frac{\left(\dot{X} \cdot X^{\prime}\right) \dot{X}^{B}-(\dot{X})^{2}\left(X^{\prime}\right)^{B}}{\sqrt{\left(\dot{X} \cdot X^{\prime}\right)^{2}-(\dot{X})^{2}\left(X^{\prime}\right)^{2}}}\right)
$$

where the various scalar products have the forms

$$
\begin{aligned}
\dot{X} \cdot X^{\prime} & =L^{2}\left\{u^{2}\left(\dot{x} x^{\prime}+\dot{r} r^{\prime}+r^{2} \dot{\phi} \phi^{\prime}\right)+\frac{1}{u^{2}}\left(\dot{\rho} \rho^{\prime}+\rho^{2} \dot{\theta} \theta^{\prime}+\dot{y}_{6} y_{6}^{\prime}\right)\right\} \\
(\dot{X})^{2} & =L^{2}\left\{u^{2}\left(-1+\dot{x}^{2}+\dot{r}^{2}+r^{2} \dot{\phi}^{2}\right)+\frac{1}{u^{2}}\left(\dot{\rho}^{2}+\rho^{2} \dot{\theta}^{2}+\dot{y}_{6}^{2}\right)\right\} \\
\left(X^{\prime}\right)^{2} & =L^{2}\left\{u^{2}\left(\left(x^{\prime}\right)^{2}+\left(r^{\prime}\right)^{2}+r^{2}\left(\phi^{\prime}\right)^{2}\right)+\frac{1}{u^{2}}\left(1+\left(\rho^{\prime}\right)^{2}+\rho^{2}\left(\theta^{\prime}\right)^{2}+\left(y_{6}^{\prime}\right)^{2}\right)\right\}
\end{aligned}
$$

and we have rewritten $y^{6}$ as $y_{6}$ to avoid confusing superscripts. The energy and momentum densities of the string are

$$
\pi_{A}^{0}=\frac{\partial \mathcal{L}}{\partial \dot{X}^{A}}=-\frac{1}{2 \pi \alpha^{\prime}} g_{A B} \frac{\left(\dot{X} \cdot X^{\prime}\right)\left(X^{\prime}\right)^{B}-\left(X^{\prime}\right)^{2}(\dot{X})^{B}}{\sqrt{\left(\dot{X} \cdot X^{\prime}\right)^{2}-\left(X^{\prime}\right)^{2}(\dot{X})^{2}}}
$$

while the energy and momentum currents read

$$
\pi_{A}^{1}=\frac{\partial \mathcal{L}}{\partial\left(X^{\prime}\right)^{A}}=-\frac{1}{2 \pi \alpha^{\prime}} g_{A B} \frac{\left(\dot{X} \cdot X^{\prime}\right)(\dot{X})^{B}-(\dot{X})^{2}\left(X^{\prime}\right)^{B}}{\sqrt{\left(\dot{X} \cdot X^{\prime}\right)^{2}-\left(X^{\prime}\right)^{2}(\dot{X})^{2}}} .
$$

We will apply Neumann boundary conditions in the D7 brane directions at $y=y_{l}$ and $y=y_{h}$

$$
\left.\pi_{A}^{1}\right|_{y=y_{h}, y_{l}}=0
$$

for $A=x, r, \phi, \rho$, and $\theta$, implying that no momentum is assumed to flow into the string from the D7 brane in these directions. The coordinate $y_{6}$ is in contrast subject to Dirichlet boundary conditions.

\section{Fluctuations in $x, \rho$ and $y_{6}$}

In this section, we study radial excitations of the heavy-light mesons. Specializing to the background of $\dot{\theta}=0, r=0$ and a constant $\rho=\rho_{0}$, we consider infinitesimal fluctuations of the string action in the form of $x=x(t, y), \rho(t, y)=\rho_{0}+\delta \rho(t, y)$ and $y_{6}=y_{6}(t, y)$. Applying eqs. (3.6)-(3.8) where now $u^{2}=y^{2}+\left(\rho_{0}+\delta \rho\right)^{2}$, we expand the action to second order in the fluctuations, and obtain

$$
\begin{gathered}
S_{\mathrm{NG}}=\frac{L^{2}}{2 \pi \alpha^{\prime}} \int d \tau d \sigma\{1 \\
-\frac{1}{2} \dot{x}^{2}+\frac{1}{2} u_{0}^{4}\left(x^{\prime}\right)^{2}+\frac{1}{2}\left(\left(\delta \rho^{\prime}\right)^{2}+\left(y_{6}^{\prime}\right)^{2}\right) \\
\left.-\frac{1}{2 u_{0}^{4}}\left(\delta \dot{\rho}^{2}+\dot{y}_{6}^{2}\right)\right\},
\end{gathered}
$$

with $u_{0}^{2} \equiv y^{2}+\rho_{0}^{2}$. 
Translational symmetry in the Minkowski directions guarantees that a constant value of $x$ is a solution to the equation of motion and thus that there is a zero mode in the spectrum corresponding to motion of the string at constant velocity in the $x$ direction. Perhaps surprisingly, a constant value of $\rho$ is also a solution and thus there is another zero mode in the spectrum corresponding to translations of the $\rho$ coordinate, even though we do not have translational symmetry in these directions. (We should emphasize that a constant value of $\rho$ is a solution to the full equations of motion (3.5) and not just to the equations of motion following from the quadratic action (4.1).) However, we will see below that this zero mode is only present for the ground state string. The fluctuating string can lower its energy by moving to $\rho=0$, a fact which strongly suggests that this time independent constant $\rho$ solution is unstable with respect to small perturbations.

There exists an interesting relationship between the equation of motion for the fluctuations in the $y_{6}$ and $\delta \rho$ directions and the equation of motion for the fluctuations in $x$ which we believe may be a consequence of supersymmetry. We will assume that the fluctuations have the time dependence $X^{A} \sim e^{-i \omega t}$ so that $\ddot{X}^{A}=-\omega^{2} X^{A}$. The equations of motion thus become

$$
\begin{aligned}
\frac{\partial}{\partial y}\left(f(y) x^{\prime}\right) & =-\omega^{2} x, \\
f(y) \delta \rho^{\prime \prime} & =-\omega^{2} \delta \rho, \quad \text { and } \quad f(y) y_{6}^{\prime \prime}=-\omega^{2} y_{6},
\end{aligned}
$$

where $f(y)=\left(y^{2}+\rho_{0}^{2}\right)^{2}$. From these expressions, it is clear that if we have a solution $x$ to eq. (4.2), then $\delta \rho=f(y) x^{\prime}$ (or $y_{6}=f(y) x^{\prime}$ ) satisfies eq. (4.3). Moreover, given a solution $\delta \rho$ (or $y_{6}$ ) to eq. (4.3), then $x=\delta \rho^{\prime}$ (or $x=y_{6}^{\prime}$ ) satisfies eq. (4.2).

A consideration of boundary conditions now reveals that the fluctuations in $x$ and $y_{6}$ have the same spectrum up to a zero mode. While $x$ and $\delta \rho$ satisfy Neumann boundary conditions, $y_{6}$ satisfies Dirichlet boundary conditions. If we solve eq. (4.2) for the allowed fluctuation modes $x$ satisfying Neumann boundary conditions, then the relations between the two equations of motion give us all the fluctuation modes $y_{6}$ satisfying Dirichlet boundary conditions. We have to perform a separate calculation for the $\delta \rho$ fluctuations, but had the $x$ fluctuations satisfied Dirichlet boundary conditions instead of Neumann, they would, too, be trivially related to the $\delta \rho$ fluctuations. We begin with the $x$ fluctuations.

\subsection{The $x$ fluctuations}

The equation (4.2) for the $x$ fluctuations can be solved to yield

$$
\begin{array}{r}
x(t, y)=\frac{C \rho_{0}}{\sqrt{y^{2}+\rho_{0}^{2}}}\left\{\sqrt{1+\frac{\omega^{2}}{\rho_{0}^{2}}} \cos \left[\sqrt{1+\frac{\omega^{2}}{\rho_{0}^{2}}} \arctan \left[\frac{y}{\rho_{0}}\right]+\alpha\right]\right. \\
\left.+\frac{y}{\rho_{0}} \sin \left[\sqrt{1+\frac{\omega^{2}}{\rho_{0}^{2}}} \arctan \left[\frac{y}{\rho_{0}}\right]+\alpha\right]\right\} e^{-i \omega t},
\end{array}
$$

where $C$ and $\alpha$ are the two integration constants. We now apply Neumann boundary conditions $x^{\prime}\left(y_{l}\right)=x^{\prime}\left(y_{h}\right)=0$ to determine the allowed spectrum $\omega$. Doing this at the 
light D7 brane, we find

$$
\alpha=-\sqrt{1+\frac{\omega^{2}}{\rho_{0}^{2}}} \arctan \left[\frac{y_{l}}{\rho_{0}}\right],
$$

while applying the boundary conditions at the heavy brane then yields the discrete spectrum:

$$
\omega_{n}^{x}=\rho_{0} \sqrt{\frac{n^{2} \pi^{2}}{\left(\arctan \left[\rho_{0} / y_{l}\right]-\arctan \left[\rho_{0} / y_{h}\right]\right)^{2}}-1}
$$

where $n \in \mathbb{Z}^{+}$. In addition to these values of $n$ however, the spectrum also contains a zero mode, the trivial solution of $\omega=0$.

Before moving onto the $y_{6}$ fluctuations, we note that in the $\rho_{0}=0$ limit, the mode functions and spectrum become simpler:

$$
\begin{aligned}
x & =C\left(\omega z \cos \left(\omega\left(z-z_{l}\right)\right)-\sin \left(\omega\left(z-z_{l}\right)\right)\right) e^{-i \omega t}, \\
\omega_{n}^{x} & =\frac{\pi n}{z_{l}-z_{h}}, \quad \text { where } \quad z=1 / y .
\end{aligned}
$$

The frequencies are the same as those of a guitar string of length $z_{l}-z_{h}$, and we thus see that in the heavy quark limit, $z_{h} \rightarrow 0$, the frequencies become $m_{h}$ independent.

\subsection{The $y_{6}$ fluctuations}

The solution to the equation of motion (4.3) is now related in a trivial way to the $x$ fluctuations studied above:

$$
y_{6}=\left(y^{2}+\rho_{0}^{2}\right)^{2} x^{\prime}=-C \omega^{2} \sqrt{\rho_{0}^{2}+y^{2}} \sin \left[\sqrt{1+\frac{\omega^{2}}{\rho_{0}^{2}}} \arctan \left[\frac{y}{\rho_{0}}\right]+\alpha\right] e^{-i \omega t} .
$$

In the $\rho_{0}=0$ limit, the mode function again takes a simpler form

$$
y_{6}=\frac{C \omega^{2}}{z} \sin \left(\omega\left(z-z_{l}\right)\right) e^{-i \omega t} \quad \text { where } \quad z=1 / y .
$$

The Dirichlet boundary conditions $y_{6}\left(y_{l}\right)=0=y_{6}\left(y_{h}\right)$ are equivalent to the Neumann boundary conditions applied to the $x$ fluctuations above, leading to the same value of $\alpha$ given in eq. (4.5) and the same spectrum

$$
\omega_{n}^{y}=\rho_{0} \sqrt{\frac{n^{2} \pi^{2}}{\left(\arctan \left[\rho_{0} / y_{l}\right]-\arctan \left[\rho_{0} / y_{h}\right]\right)^{2}}-1},
$$

where $n \in \mathbb{Z}^{+}$. This time, however, there is no zero mode.

\subsection{The $\delta \rho$ fluctuations}

For the $\delta \rho$ fluctuations, we will not be able to find an analytic spectrum, but will eventually attempt to understand the spectrum's features both qualitatively and numerically. We begin with the general solution to eq. (4.3),

$$
\delta \rho(t, y)=C \sqrt{\rho_{0}^{2}+y^{2}} \sin \left[\sqrt{1+\frac{\omega^{2}}{\rho_{0}^{2}}} \arctan \left[\frac{y}{\rho_{0}}\right]+\alpha\right] e^{-i \omega t} .
$$


Applying Neumann boundary conditions at the light brane $\delta \rho^{\prime}\left(y_{l}\right)=0$, we find

$$
\alpha=-\sqrt{1+\frac{\omega^{2}}{\rho_{0}^{2}}} \arctan \left[\frac{y_{l}}{\rho_{0}}\right]-\arctan \left[\sqrt{1+\frac{\omega^{2}}{\rho_{0}^{2}}} \frac{\rho_{0}}{y_{l}}\right],
$$

while demanding that the boundary conditions are satisfied at the heavy brane leads to

$$
\tan \left[\sqrt{1+\frac{\omega^{2}}{\rho_{0}^{2}}}\left(\arctan \left[\frac{y_{l}}{\rho_{0}}\right]-\arctan \left[\frac{y_{h}}{\rho_{0}}\right]\right)+\arctan \left[\sqrt{1+\frac{\omega^{2}}{\rho_{0}^{2}}} \frac{\rho_{0}}{y_{l}}\right]\right]=\sqrt{1+\frac{\omega^{2}}{\rho_{0}^{2}}} \frac{\rho_{0}}{y_{h}} .
$$

The solutions of this equation give us the spectrum of the fluctuations $\omega_{n}^{\rho}$.

Unfortunately, the transcendental nature of the above equation prevents us from solving it analytically. There are, however, various limits, where we can simplify the numerical solution. The first simplification occurs in the limit of large $y_{h}$, in which one may attempt a power expansion in $y_{l} / y_{h}$. To this end, we write

$$
\omega_{n}^{\rho} \equiv \omega_{n}=y_{l} \times \sum_{i=0}^{\infty} \omega_{n, i}\left(\frac{y_{l}}{y_{h}}\right)^{i}
$$

substitute this into eq. 4.14, and proceed to solve the equation order by order in the small parameter $y_{l} / y_{h}$. At leading order, we easily obtain for $\omega_{n, 0}$

$$
\sqrt{1+\frac{\omega_{n, 0}^{2} y_{l}^{2}}{\rho_{0}^{2}}}\left(\frac{\pi}{2}-\operatorname{arccot}\left[\frac{\rho_{0}}{y_{l}}\right]\right)-\arctan \left[\sqrt{1+\frac{\omega_{n, 0}^{2} y_{l}^{2}}{\rho_{0}^{2}}} \frac{\rho_{0}}{y_{l}}\right]=n \pi,
$$

with $n \in \mathbb{Z}^{+}$. The numerical solution to this equation quickly leads to the forms of the functions $\omega_{n, 0}\left(\rho_{0} / y_{l}\right)$. The next two terms in the power series expansion of eq. (4.14) are solved trivially by setting $\omega_{n, 1}$ and $\omega_{n, 2}$ equal to zero, and it is only at order $i=3$ that we find the next nonzero term in the expansion of eq. 4.15). The forms of the resulting functions $\omega_{n, 0}\left(\rho_{0} / y_{l}\right)$ and $\omega_{n, 3}\left(\rho_{0} / y_{l}\right)$ will be displayed for $n=1,2, \ldots, 5$ in the next section in a slightly different notation.

One limit, where the functions $\omega_{n, i}$ are in fact analytically solvable is that of large $\rho_{0} / y_{l}$. There, it is straightforward to see that eq. (4.16) reduces to the solution

$$
\omega_{n, 0}=\sqrt{(2 n+1)^{2}-1} \frac{\rho_{0}}{y_{l}},
$$

while the three next orders produce

$$
\omega_{n, 1}=\omega_{n, 2}=0 \quad \text { and } \quad \omega_{n, 3}=\frac{4}{3 \pi} \sqrt{n(n+1)}(2 n+1)^{2}\left(\frac{\rho_{0}}{y_{l}}\right)^{4} .
$$

It is interesting to contrast eq. (4.17) with the spectra of the $x$ and $y_{6}$ fluctuations, which in the same limit $\left(y_{h} \rightarrow \infty\right.$ and $\rho_{0} / y_{l}$ large) produce from eq. (4.6)

$$
\omega_{n}^{x}=\sqrt{(2 n)^{2}-1} \rho_{0} .
$$


We thus see that at least in this limit, the fluctuation energies in the $x$ and $y_{6}$ direction lie exactly in between the energies of the $\rho$ fluctuations.

Finally, we note that in the limit $\rho_{0}=0$, eq. (4.12) reduces to

$$
\delta \rho=\frac{-C}{z \sqrt{1+\omega^{2} z_{l}^{2}}}\left(\omega z_{l} \cos \left(\omega\left(z-z_{l}\right)\right)+\sin \left(\omega\left(z-z_{l}\right)\right)\right) e^{-i \omega t},
$$

while condition (4.14) on the frequencies reduces to the simple expression

$$
\omega\left(z_{h}-z_{l}\right)=\arctan \left(\omega z_{h}\right)-\arctan \left(\omega z_{l}\right)-\pi n,
$$

where $n$ is an integer. This equation, however, is not of an analytically solvable type either, so it must be dealt with numerically. In the limit $y_{h} \rightarrow \infty$, the first few solutions are $\omega z_{l}=4.493,7.725$, and 10.904 .

\subsection{The meson mass spectrum}

Let us finally look at the energy spectrum of the string fluctuations in more detail. Using the result

$$
E=-\int \mathrm{d} \sigma \pi_{t}^{0}
$$

we see that to quadratic order in the fluctuations the energy of the string can be obtained by integrating the canonical momentum density

$$
\pi_{t}^{0}=-\frac{L^{2}}{2 \pi \alpha^{\prime}}\left(1+\frac{1}{2} u^{4}\left(x^{\prime}\right)^{2}+\frac{1}{2} \dot{x}^{2}+\frac{1}{2}\left(\delta \rho^{\prime}\right)^{2}+\frac{1}{2 u^{4}}(\delta \dot{\rho})^{2}+\frac{1}{2}\left(y_{6}^{\prime}\right)^{2}+\frac{1}{2 u^{4}}\left(\dot{y}_{6}\right)^{2}\right) .
$$

From a classical perspective, the energies will depend on the amplitudes of the fluctuations, while from a quantum perspective, these amplitudes can only take on discrete values corresponding to the occupation number of a given mode. At quadratic order, we essentially have a version of the quantum harmonic oscillator. The equal time commutation relation $\left[X^{A}(y), \pi_{A}^{0}\left(y^{\prime}\right)\right]=i \delta\left(y-y^{\prime}\right)$ implies, in units where $\hbar=1$, that the smallest quanta of excitation are the frequencies we determined before, the $\omega_{n}^{w}$ where $w=x, \rho$, or $y$. We find the simple result

$$
E=m_{h}-m_{l}+\sum_{w, n} N_{w}^{n} \omega_{n}^{w}
$$

where $N_{w}^{n}$ is the occupation number of the mode $(w, n) .{ }^{5}$ We therefore note that in order to inspect the mass spectrum of the heavy-light mesons below, we merely need to consider the frequencies $\omega_{n}^{w}$ obtained above. We anticipate eq. (4.23) remains valid provided $N_{w}^{n} \ll \sqrt{\lambda}$ and we can neglect the nonlinearities in the string equation of motion. Consistent with the observation that a constant nonzero $\rho_{0}$ is a solution to the full equations of motion, note that in the absence of fluctuations, the energy of the string is $m_{h}-m_{l}$ for all $\rho_{0}$.

\footnotetext{
${ }^{5}$ Calculating the zero point energy contribution to these oscillators requires also investigating the fermionic fluctuations of the superstring. We suspect supersymmetry implies that the zero point energy vanishes.
} 
The $\boldsymbol{x}$ and $\boldsymbol{y}_{6}$ fluctuations. Denoting $q \equiv \rho_{0} L^{2} / 2 \pi \alpha^{\prime}$ and using the relation $L^{2} / \alpha^{\prime}=$ $\sqrt{\lambda}$, we can write the energy spectrum of the $x$ or $y_{6}$ fluctuations in the form

$$
E_{n}^{x}=E_{n}^{y}=m_{h}-m_{l}+\frac{2 \pi q}{\sqrt{\lambda}} \sqrt{\frac{n^{2} \pi^{2}}{\left(\arctan \left[q / m_{l}\right]-\arctan \left[q / m_{h}\right]\right)^{2}}-1} .
$$

This formula gives the energy for a string with a single quantum of excitation in the $n$th mode of the $y_{6}$ or $x$ fluctuations. In the Introduction, we claimed that in the heavy quark limit, $m_{h} \gg m_{l}$, the energy of the excitations scaled with $m_{l}$. Here, seemingly in contradiction with the earlier claim, we find that in the limit $m_{h} \gg q$, we may expand the $\omega_{n}^{x}$ in inverse powers of $m_{h}$, producing

$$
E_{n}^{x}=m_{h}-m_{l}+\frac{2 \pi q}{\sqrt{\lambda}} g_{n}\left(\frac{q}{m_{l}}\right)+\frac{2 \pi^{3} n^{2} q^{2}}{\sqrt{\lambda} m_{h}} \frac{1}{\arctan ^{3}\left[q / m_{l}\right] g_{n}\left(q / m_{l}\right)}+\mathcal{O}\left(\frac{1}{m_{h}^{2}}\right)
$$

where we have denoted

$$
g_{n}(x) \equiv \sqrt{\frac{n^{2} \pi^{2}}{\arctan ^{2}[x]}-1}
$$

Thus, the excitation spectrum depends on both light scales $m_{l}$ and $q$.

We now give two reasons why the scale $q$ should disappear. First, the derivative of the excitation energies with respect to $q$ is non-negative

$$
\frac{\partial E_{n}^{x}}{\partial q}=\frac{\partial E_{n}^{y}}{\partial q} \geq 0
$$

and is equal to zero at $q=0$, implying that fluctuations about $q \neq 0$ have more energy than the equivalent fluctuations about $q=0$. This inequality suggests that a string initially fluctuating about a nonzero value of $\rho_{0}$ will experience a potential that will cause it to start oscillating about $\rho=0$. In the case of $q=0$, the energy spectra reduce to

$$
E_{n}^{x}=E_{n}^{y}=m_{h}-m_{l}+\frac{m_{h} m_{l}}{m_{h}-m_{l}} \frac{2 \pi^{2} n}{\sqrt{\lambda}}
$$

where $n \in \mathbb{Z}^{+}$. In the heavy quark limit $m_{h} \gg m_{l}$, the excitation spectrum does indeed depend only on $m_{l}$ to leading order in $m_{l} / m_{h}$.

The second reason for the disappearance of the scale $q$ will be developed more in section 5, where we will see that for slowly spinning strings in the $\rho-\theta$ plane, a nonzero value of $\rho_{0}$ is stabilized. Thus what would seem to be a zero mode in the $\rho$ direction is lifted and a continuous change of $q$ will not be possible for these spinning strings. However, the stable value of $\rho_{0}$ is of order $m_{l}$ or zero, regardless of the angular momentum, and thus the extra scale $q$ again disappears from the excitation spectrum.

The $\delta \rho$ fluctuations. For the $\delta \rho$ fluctuation spectrum given by eq. 4.14, we have to resort to numerics. In the limit of large $y_{h} \gg y_{l}$, we may use our earlier numerical solution 

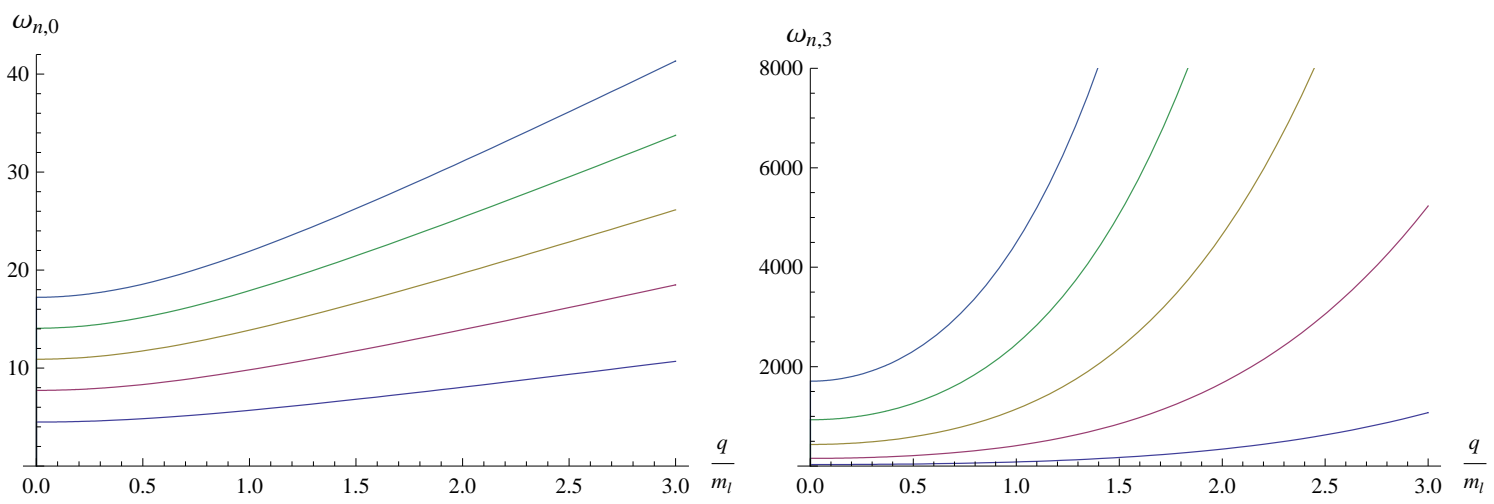

Figure 2: Plots of the functions $\omega_{n, 0}\left(q / m_{l}\right)$ and $\omega_{n, 3}\left(q / m_{l}\right)$, respectively. The index $n$ grows from 1 to 5 from the bottom to the top curve in both figures.

utilizing a power series expansion in $y_{l} / y_{h}$, in terms of which the spectrum can be written in the form

$$
E_{n}^{\rho}=m_{h}-m_{l}+m_{l} \omega_{n, 0}\left(q / m_{l}\right) \frac{2 \pi}{\sqrt{\lambda}}+\frac{m_{l}^{4}}{m_{h}^{3}} \omega_{n, 3}\left(q / m_{l}\right) \frac{2 \pi}{\sqrt{\lambda}}+\mathcal{O}\left(m_{l}^{5} / m_{h}^{4}\right)
$$

This formula corresponds to the energy of a string with a single quantum of energy in the $n$th mode of the $\rho$ fluctuations. We plot the functions $\omega_{n, 0}$ and $\omega_{n, 3}$ in figure 2 . From there, we see that the energies of the fluctuations are always minimized at $\rho_{0}=0$ or $q=0$, just as it was for the $x$ and $y_{6}$ fluctuations. Another interesting aspect of these excitation energies is the absence of the two first leading corrections in $m_{l} / m_{h}$ in the heavy quark limit.

\section{Spinning strings}

To supplement our discussion of the small fluctuations of strings around static quarkantiquark solutions, we now turn to consider the case where the string joining the heavy and the light brane is spinning. First, we consider strings spinning in the real space where they have a conserved angular momentum, and then look into strings spinning in the internal $\theta$ direction where the corresponding angular momentum can be reinterpreted as a charge. Our analysis is purely classical, but we expect valid, provided the angular momentum and charge of the strings are large.

As we have discussed briefly already, there is an interesting wrinkle in the discussion of the $\rho-\theta$ spinning strings. A straight, motionless string stretching between the D7 branes at a nonzero value of $\rho_{0}$ is a solution for all $\rho_{0}$. That such a string is a solution is surprising given the lack of translation invariance in $\rho$. As we saw before in the analysis of the fluctuations, if we excite one of these straight strings with $\rho_{0} \neq 0$, it will experience a force pulling it toward $\rho=0$. In this section on spinning strings, we will find that a string spinning in the $\rho-\theta$ plane is not free to sit at an arbitrary average value of $\rho_{0}$ either. 


\subsection{Strings spinning in real space}

We start by looking into the profile and energy spectrum of a string spinning in real space, more specifically in the $x^{1}-x^{2}$ plane, assuming that $x^{3}=\rho=y_{6}=0$. To begin with, we transform from Cartesian $\left(x^{1}, x^{2}\right)$ to polar coordinates $(r, \phi)$, and make the uniformly rotating ansatz of ref. [12], where $\phi=\Omega t$ is independent of the worldsheet coordinate $\sigma{ }^{6}$ At the same time, we assume that $z(\sigma)$ and $r(\sigma)$ are $t$ independent, which leads to an action of the form

$$
S=-\frac{L^{2}}{2 \pi \alpha^{\prime}} \int d t d \sigma \frac{1}{z^{2}} \sqrt{\left(1-\Omega^{2} r^{2}\right)\left(\left(z^{\prime}\right)^{2}+\left(r^{\prime}\right)^{2}\right)},
$$

invariant under reparametrizations of the worldsheet coordinate $\sigma=f\left(\sigma^{\prime}\right)$. For the most part, we will choose $\sigma=z$, though for the numerical studies we will shortly present, we found it sometimes convenient to make other choices, such as $\sigma=r$. This action leads to the following formulae for the energy and angular momentum of the string:

$$
\begin{aligned}
& E=\frac{L^{2}}{2 \pi \alpha^{\prime}} \int d \sigma \frac{1}{z^{2}} \sqrt{\frac{\left(z^{\prime}\right)^{2}+\left(r^{\prime}\right)^{2}}{1-\Omega^{2} r^{2}}}, \\
& J=\frac{L^{2} \Omega}{2 \pi \alpha^{\prime}} \int d \sigma \frac{r^{2}}{z^{2}} \sqrt{\frac{\left(z^{\prime}\right)^{2}+\left(r^{\prime}\right)^{2}}{1-\Omega^{2} r^{2}}} .
\end{aligned}
$$

Choosing now $\sigma=r$, the equation of motion for $r(z)$ has the form

$$
\frac{r^{\prime \prime}}{1+\left(r^{\prime}\right)^{2}}-\frac{2}{z} r^{\prime}+\frac{\Omega^{2} r}{1-\Omega^{2} r^{2}}=0
$$

which we now proceed to solve, demanding that Neumann boundary conditions be satisfied on the heavy and light branes at $z=z_{h}$ and $z=z_{l}$. Neumann boundary conditions for $\phi$ are satisfied trivially because $\phi^{\prime}=0$, while for $r$ the boundary conditions read

$$
\left.r^{\prime} \sqrt{\frac{1-\Omega^{2} r^{2}}{1+\left(r^{\prime}\right)^{2}}}\right|_{z=z_{h}, z_{l}}=0 .
$$

Thus, we must either require that $r^{\prime}=0$ at the boundary or that $\Omega^{2} r^{2}=1$, which physically is the condition that the endpoint of the string is moving at the local speed of light. We will in general choose $r^{\prime}=0$, but will nevertheless find certain "critical" solutions that satisfy the light-like boundary conditions.

The linearized form of eq. (5.4) provides a good place to begin our study, as this form

$$
z^{2}\left(\frac{r^{\prime}}{z^{2}}\right)^{\prime}+\Omega^{2} r=0
$$

of eq. (5.4), valid when $r^{\prime}$ and $\Omega r \ll 1$, is easy to solve. Indeed, we already solved it; eq. (5.6) is identical to eq. (4.2) in the case $\rho_{0}=0$. Assuming then that the string takes the form

$$
\begin{aligned}
& r=A\left(\omega_{n} z \cos \left(\omega_{n}\left(z-z_{l}\right)\right)-\sin \left(\omega_{n}\left(z-z_{l}\right)\right)\right), \\
& \phi=\omega_{n} t=\frac{\pi n}{z_{l}-z_{h}} t
\end{aligned}
$$

\footnotetext{
${ }^{6}$ We have checked that this ansatz satisfies the full equations of motion (3.5).
} 


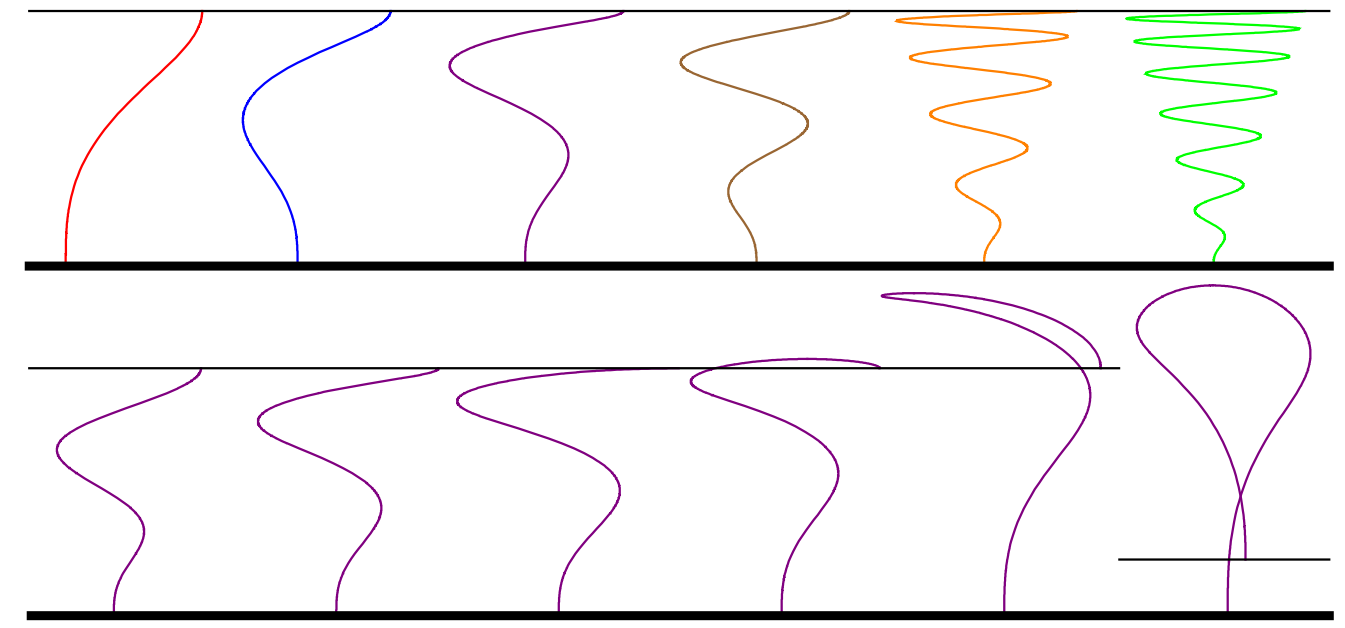

Figure 3: Top: A schematic plot showing the forms of the spinning string solutions $r(z)$ for various $n$. The lower (thick) horizontal line corresponds to the heavy brane sitting at $z_{h}=1 / 100$ and the upper (thin) line to the light brane at $z_{l}=1$, with the coordinate $z$ growing vertically. The six curves, from left to right, correspond to the cases of $n=1,2,3,4,9$ and 13, respectively. Bottom: Another schematic plot showing the evolution of the $n=3$ branch as $\Omega$ is decreased from 9.52 (left) to 0.5 (right). The critical solution $\Omega_{3 c}=5.84$ is the third from the left. For the smallest value of $\Omega$, corresponding to large $J$ and $E$, we have rescaled the solution in the $z$ direction by a factor of 4.4 in order to make it fit in the figure. In the $\Omega \rightarrow 0$ limit, the solution becomes symmetric in the $r$ direction about the center of mass.

for small $A$, where we have adapted eq. (4.7), the energy and angular momentum are given by the approximate expressions

$$
\begin{aligned}
E & =\frac{L^{2}}{2 \pi \alpha^{\prime}}\left(\frac{1}{z_{h}}-\frac{1}{z_{l}}-\frac{(\pi n)^{4} A^{2}}{2\left(z_{h}-z_{l}\right)^{3}}+\mathcal{O}\left(A^{4}\right)\right), \\
J & =\frac{L^{2}}{2 \pi \alpha}\left(\frac{(\pi n)^{3} A^{2}}{2\left(z_{h}-z_{l}\right)^{2}}+\mathcal{O}\left(A^{4}\right)\right) .
\end{aligned}
$$

Eliminating $A$ from here, we find that ${ }^{7}$

$$
E \approx m_{h}-m_{l}+n \pi \frac{m_{l} m_{h}}{m_{h}-m_{l}} \frac{2 \pi J}{\sqrt{\lambda}}
$$

which corresponds to the dashed straight lines in figure 1 (left), where we display the $E$ vs. $J$ dependence of our spinning strings. This linear scaling of $E$ with $J$ is characteristic of a particle in a Hooke's law potential, where the constant of proportionality is given by the frequency of the oscillator. ${ }^{8}$

As the $E$ and $J$ of the string get larger, $r$ will get larger as well, and eventually our linearized approximation breaks down. To make further progress, we resort to numerics to

\footnotetext{
${ }^{7}$ The $n=1$ version of this formula $(\sqrt[5.11]{ })$ was first presented in ref. 114.

${ }^{8}$ In general, for a nonrelativistic particle in a central force potential $\mathrm{U}(r)=c r^{a}$, one finds that $E \sim$ $L^{2 a /(2+a)}$ where for us the relevant angular momentum $L$ is either $J$ or $Q$.
} 

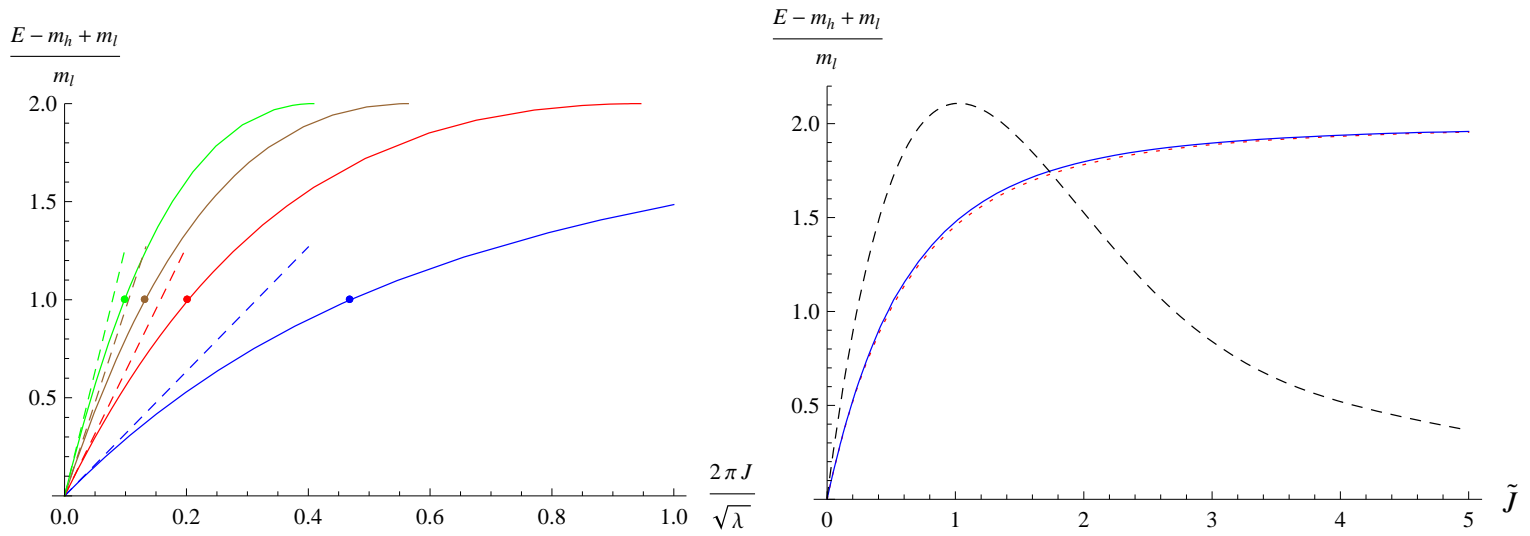

Figure 4: Left: The dependence of $E$ versus $J$ for the spinning heavy-light mesons. We display the curves for $m_{h}=100 m_{l}$ and $n=1,2,3,4$ from right to left, with the adjacent dashed straight lines corresponding to the respective analytic small- $J$ approximations of eq. (5.11) and the dots on the curves denoting the critical solutions at $\Omega=\Omega_{\mathrm{nc}}$. Right: The $E(\tilde{J})$ curves for both the $m_{h}=100 m_{l}$ (solid blue curve) and $m_{h}=10 m_{l}$ (dotted red) cases for the $n=1$ branch, together with their difference multiplied by a factor of 100 (dashed black).

calculate the profile $(r, z)$ of the spinning strings. For simplicity, we rescale our variables so that $z_{l}=1$, and have $z_{h}$ take the values $1 / 10$ and $1 / 100$, corresponding roughly to the heavy-to-light quark mass ratios one finds in QCD for charm and bottom quarks. We find that for each $n$, there is a continuous family of rotating string solutions for all $\Omega$ such that $0<\Omega<\omega_{n}$. The index $n$ parametrizes the number of turning points in the solutions: For the branch $n$, the string profile $(r, z)$ has always $n-1$ (local) extremal values in $r$. Examples of the profile $(r, z)$ for various $n$ are exhibited in figure 3 (top).

Once the results for $(r, z)$ are obtained in a numerical form, we insert them into the integrals of eqs. (5.2) and (5.3), thus obtaining the energies of the spinning strings in terms of their angular momenta. The resulting curves $f_{n}(x)$, parametrizing the energies through

$$
E_{n}=m_{h}-m_{l}+m_{l} f_{n}(2 \pi J / \sqrt{\lambda})
$$

are shown for $n=1,2,3,4$ and $z_{h}=1 / 100$ in figure 4 (left) and in more detail for the $n=1$ branch in figure 5. Intriguingly, reducing $\Omega$ increases both $E$ and $J$. A similar behavior was observed for the heavy-heavy mesons in ref. [12], and is explained by the fact that the decrease in $\Omega$ is made up for by the growing size of the string. The evolution of the profile of the $n=3$ branch string as a function of $\Omega$ is shown in figure 3 (bottom).

The dependence of the $E(J)$ curves on $m_{h}$ is relatively mild and easily modeled. The eq. (5.11) suggests a rescaling of the variable $J$ by $1 /\left(1-m_{l} / m_{h}\right)$, defining

$$
\tilde{J}=\frac{m_{h}}{m_{h}-m_{l}} \frac{2 \pi J}{\sqrt{\lambda}} .
$$

With this small correction, we see from figure 1 (right) that the curves corresponding to $z_{h}=1 / 10$ and $1 / 100$ practically overlap. 


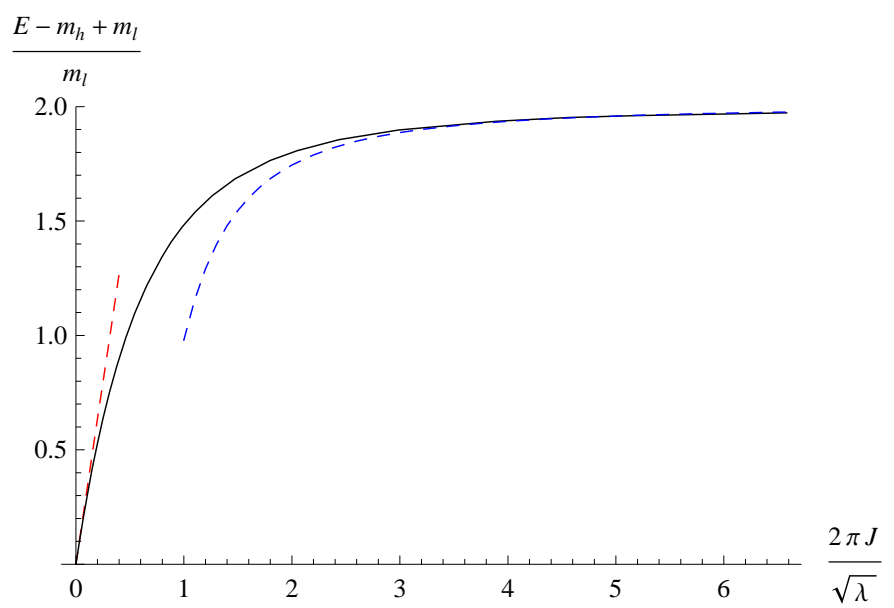

Figure 5: We plot $E$ versus $J$ for the $n=1$ branch of the spinning heavy-light mesons. The solid curve is the numerical result for the case $m_{h}=100 m_{l}$, while the red and blue dashed curves are the analytic small and large- $J$ approximations of eqs. (5.11) and (5.16), respectively.

As $\Omega$ is decreased, there is a critical $\Omega_{\mathrm{nc}}$ for each family of solutions where the light quark endpoint of the string is moving at the local speed of light, $\Omega_{\mathrm{nc}} r\left(z_{l}\right)=1$. For the short strings with $\Omega>\Omega_{\mathrm{nc}}$, the string is contained entirely between the two D7 branes, while for the long strings with $\Omega<\Omega_{\mathrm{nc}}$, there is a loop of string in the region $z>z_{l}$. Like the $\omega_{n}$, the critical $\Omega_{\mathrm{nc}}$ depend to some extent on the choice of the heavy and light quark masses. For the first few $n$, we find that

$$
\begin{aligned}
& z_{h}=1 / 10: \quad \Omega_{1 c}=1.54, \quad \Omega_{2 c}=3.98, \quad \Omega_{3 c}=6.22, \quad \Omega_{4 c}=8.41, \\
& z_{h}=1 / 100: \quad \Omega_{1 c}=1.38, \quad \Omega_{2 c}=3.72, \quad \Omega_{3 c}=5.84, \quad \Omega_{4 c}=7.91 \text {. }
\end{aligned}
$$

We furthermore observe that for $n=1$, the critical energies and angular momenta obey the results

$$
\begin{aligned}
E_{c} & =m_{h}-\frac{m_{l}^{2}}{2 m_{h}}+\mathcal{O}\left(\frac{m_{l}^{3}}{m_{h}^{2}}\right), \\
\tilde{J}_{c} & =0.473-0.262 \frac{m_{l}}{m_{h}}+\mathcal{O}\left(\frac{m_{l}^{2}}{m_{h}^{2}}\right),
\end{aligned}
$$

and that for $n>1$, the forms of the equations stay intact, while the numbers in the latter relation somewhat change. Especially the former of these results deserves some attention; we have verified this relation to more than 1 part in 10000, but have so far no explanation for why the limiting energy should obtain such a simple form.

As $\Omega$ is decreased below $\Omega_{\mathrm{nc}}$, the strings quickly begin to get very large compared to the separation between the D7 branes, and in the $\Omega \rightarrow 0$ limit, their size in fact diverges both in the $r$ and $z$ directions. Indeed, in this limit the spinning string solutions can be seen to approach those of the heavy-heavy mesons considered in ref. [12], where both ends of the string end on the same D7 brane. The limit $\Omega \rightarrow 0$ of the $n=1$ branch is special 
because the velocity of any point on the $n=1$ string approaches zero as $\Omega$ decreases, while for the $n>1$ branches, there always exists a finite set of points $\sigma_{i}$ along the string where, due to the large size of the string, $r\left(\sigma_{i}\right) \Omega \rightarrow 1$ as $\Omega \rightarrow 0$. As noticed originally by refs. [12, 14, the small size of $\Omega r$ allows for an analytic treatment of the $E$ and $J$ of the $n=1$ branch in the $\Omega \rightarrow 0$ limit.

In the $\Omega \rightarrow 0$ limit, the strings correspond to marginally bound heavy-light mesons with an energy $E \approx m_{h}+m_{l}$. By marginally bound, we mean that the binding energy becomes very small. For the $\Omega \rightarrow 0$ limit of the $n=1$ branch, the string profile must be well approximated by the static configuration that determines the potential between two infinitely massive quarks. As shown in ref. [14], in this limit the energy of the string obeys the relation

$$
E=m_{h}+m_{l}-\kappa \frac{m_{l} m_{h}}{m_{h}+m_{l}} \frac{\lambda}{J^{2}}
$$

where

$$
\kappa=2\left(\frac{\Gamma(3 / 4)}{\Gamma(1 / 4)}\right)^{4} \approx 0.0261
$$

consistent with a Coulombic attraction between the quarks. We see from figure that eq. (5.16) is quite a good approximation to the $E(J)$ curve already at moderately large $J$. In contrast, the $\Omega \rightarrow 0$ limit of the $n>1$ branches all terminate at finite values of $J$. Numerically, for the case of $m_{h}=100 m_{l}$, these terminal values of $2 \pi J / \sqrt{\lambda}$ are 0.946 , 0.546 , and 0.409 for the $n=2,3$ and 4 branches, respectively.

We believe that the long strings are much less stable than the short strings. For one, they intersect the D7 brane and thus can break in two. For another, they are much bigger in size than the short strings, and thus it is likely that they are subject to instabilities, which do not respect the uniformly rotating $\phi=\Omega t$ ansatz.

\subsection{String profile in $\rho$ and $\theta$}

Next, we look at the profile of a string spinning inside the $\mathbb{R}^{6}$, in the $\rho-\theta$ directions. Let $Q$ be the corresponding angular momentum. Although $Q$ is an angular momentum from the ten dimensional point of view, in the four dimensional field theory it is a charge, namely the R-charge of the R-symmetry of our supersymmetric field theory. From the point of view of QCD, $Q$ could be viewed as a model of the electromagnetic charge of the meson.

To begin with, we assume that $x=r=y_{6}=0$, and in analogy with our discussion of strings spinning in real space, make an ansatz where $\rho(y)$ is time independent and $\theta=\Omega t$ is $y$ independent. ${ }^{9}$ The Neumann boundary conditions for $\theta$ are then again trivially satisfied because $\theta^{\prime}=0$. With these simplifications, the action for the string reduces to

$$
S_{\mathrm{NG}}=-\frac{L^{2}}{2 \pi \alpha^{\prime}} \int d t d y \sqrt{\left(1-\rho^{2} \Omega^{2} / u^{4}\right)\left(1+\left(\rho^{\prime}\right)^{2}\right)},
$$

leading to the equation of motion for $\rho(y)$,

$$
\frac{u^{2} \rho^{\prime \prime}}{1+\left(\rho^{\prime}\right)^{2}}+\Omega^{2} \rho \frac{u^{2}-2 \rho^{2}+2 y \rho \rho^{\prime}}{u^{4}-\Omega^{2} \rho^{2}}=0 .
$$

\footnotetext{
${ }^{9}$ We have checked that this ansatz satisfies the full equations of motion (3.5).
} 
The energy $E$ and internal angular momentum $Q$ of the spinning strings are given by

$$
\begin{aligned}
& E=\frac{L^{2}}{2 \pi \alpha^{\prime}} \int d y \sqrt{\frac{1+\left(\rho^{\prime}\right)^{2}}{1-\rho^{2} \Omega^{2} / u^{4}}}, \\
& Q=\frac{L^{2}}{2 \pi \alpha^{\prime}} \int d y \frac{\rho^{2} \Omega}{u^{4}} \sqrt{\frac{1+\left(\rho^{\prime}\right)^{2}}{1-\rho^{2} \Omega^{2} / u^{4}}} .
\end{aligned}
$$

The Neumann boundary conditions for $\rho$ on the other hand reduce to the requirement

$$
\left.\rho^{\prime} \sqrt{\frac{1-\rho^{2} \Omega^{2} / u^{4}}{1+\left(\rho^{\prime}\right)^{2}}}\right|_{y=y_{h}, y_{l}}=0
$$

from where we see that we must again either require that $\rho^{\prime}=0$ at the boundary or that the ends of the string move at the local speed of light. Similar to the strings spinning in real space, we generically enforce $\rho^{\prime}=0$, but in addition find certain special solutions that satisfy the light-like boundary conditions. Note that a motionless string with $\rho=\rho_{0}$ and $\Omega=0$ is a solution to the equations of motion for all $\rho_{0}$. Once $\Omega \neq 0$, however, the story becomes much more interesting.

For non-zero $\Omega$, the equation of motion for $\rho$, eq. (5.18), seems difficult to solve analytically at least in full generality, and we will therefore resort to numerics, setting again $y_{l}=1$ and varying the location of the heavy brane $y_{h}$. The story we encounter is strongly reminiscent of the strings spinning in real space. We again find multiple branches of solutions indexed by an integer $n, n \geq 1$, with the corresponding string profiles $\rho_{n}(y)$ containing exactly $n-1$ extrema in $\rho$.

The low energy behavior of our strings can again be understood analytically through the fluctuation analysis of the previous section. In this $E \rightarrow 0$ limit, we may take the string profiles to be complex combinations of $\rho$ fluctuations with infinitesimal amplitude. The complex combination produces a string spinning in the $\rho-\theta$ plane with angular velocity $\Omega=\omega_{n}$, corresponding to the solutions to eq. (4.21). Consistent with the results from section 4.3, we see that for $y_{h}=100$, the values of the first few $\omega_{n}$ 's are 4.493, 7.725, 10.904.

For a given $n>0$, we find a continuous family of solutions in the range $0<\Omega<\omega_{n}$. Decreasing $\Omega$ corresponds to increasing $E$ and $J$, the increase in the size of the string more than making up for the loss of angular velocity. There are again critical angular frequencies $\Omega_{\mathrm{nc}}$ which separate the long strings with $\Omega<\Omega_{\mathrm{nc}}$ from the short strings with $\Omega>\Omega_{\mathrm{nc}}$, the former extending to the region $y<y_{l}$. For the critical solution, the endpoint of the string sitting on the light brane is moving at the local speed of light. For $y_{h}=100$, the critical angular velocities for the first three branches are found to equal $\Omega_{1 c}=3.260, \Omega_{2 c}=5.152$ and $\Omega_{3 c}=7.108$.

In addition to the branches with $n \geq 1$, we find an additional branch of solutions which has no analog for the strings spinning in real space. By a small abuse of notation, we will call this branch $n=0$ although like the $n=1$ branch, it has no extrema between the endpoints of the string. This branch of the spinning strings emerges from the lifting of the zero fluctuation mode corresponding to translations in the $\rho$ direction, and as we will show 


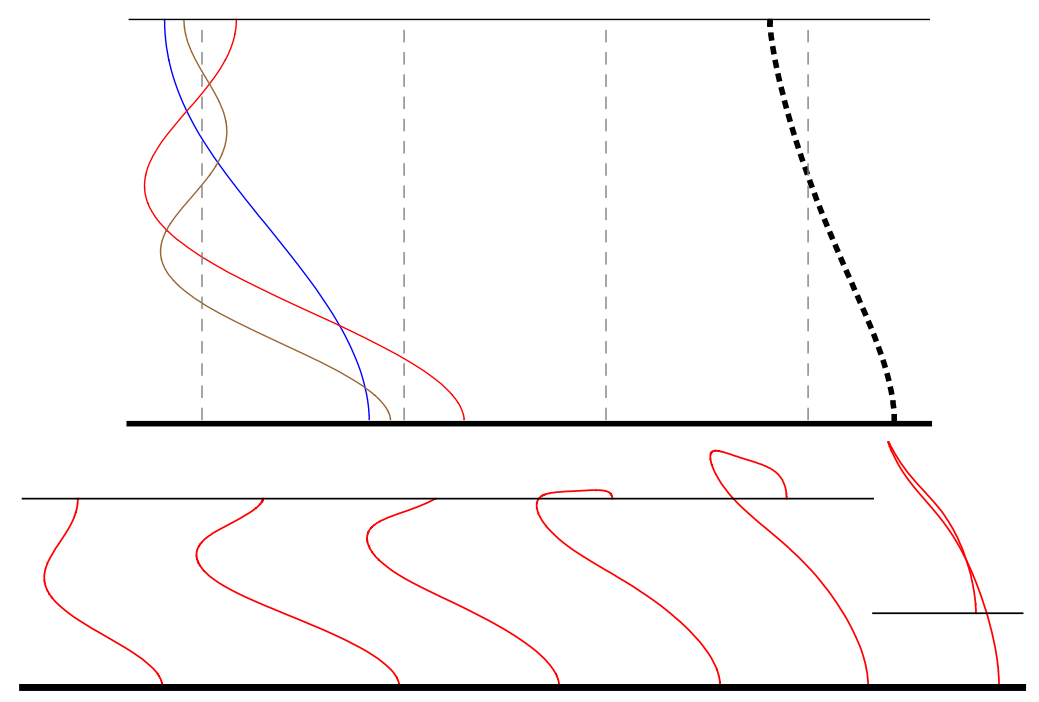

Figure 6: Top: Profiles of the spinning strings $\rho(z)$ stretching between the two branes at $z_{l}=1$ and $z_{h}=1 / 100$, with $z \equiv 1 / y$ and the notation as in figure 3 . The black dotted line corresponds to the $n=0$ branch, while the blue, red and brown solid curves correspond to the $n=1,2,3$ cases, respectively. The gray dashed lines, from left to right, mark the points $\rho=0,1 / 2,1,3 / 2$. Bottom: The evolution of the $n=2$ branch of solutions as $\Omega$ is decreased from 7.725 to 1 . The critical solution is again the third from the left, while the smallest $\Omega$ solution has been rescaled in the $z$ direction by a factor 2.55 . In the $\Omega \rightarrow 0$ limit, the part of the solution extending beyond the light brane doubles back on itself.

shortly, it is possible to understand its low-energy properties in a semi-analytic fashion. Earlier in our fluctuation analysis, we saw that while the ground state string sitting at $\rho \neq 0$ with $\Omega=0$ did not experience a potential, excited strings felt a force pulling them toward $\rho=0$. Here, we instead find that strings with even an arbitrarily small $\Omega$ are not free to move in the $\rho$ direction, but must sit at a constant $\rho=\rho_{0}$ in the limit where $\Omega$ tends to zero. We do not see an $n=0$ branch for the real space spinning strings because the zero mode in the $x^{\mu}$ directions is not lifted.

Inspecting the $n=0$ branch numerically for $y_{h}=100$, we observe that $\Omega$ can be arbitrarily close to zero, with the $\Omega \rightarrow 0$ limit corresponding to small angular momenta and energies, in contrast to the branches with $n \geq 1$. In this limit, the string profile becomes a constant, equaling $\rho(y) \equiv \rho_{0} \approx 1.825$. This time there is no maximal angular velocity at which the solution breaks down, but we rather find that the curve that this branch of solutions draws on the $\left(\Omega, \rho\left(y_{l}\right)\right)$ plane is not a single valued function of $\Omega$. For the $y_{h}=100$ case we are considering, it starts from the point $(0,1.825)$, follows monotonically to the point $(2.082,1.361)$ and finally turns back to end at $(2.069,1.300)$, where the light end of the string is spinning at the local speed of light. We exhibit the forms of the string profiles for $n=0,1,2,3$ in figure 6 .

In figure 0 , we plot the $E$ vs. $Q$ dependence of the different branches of spinning string solutions we have encountered. Let us first focus on the $n \geq 1$ branches, and specifically 

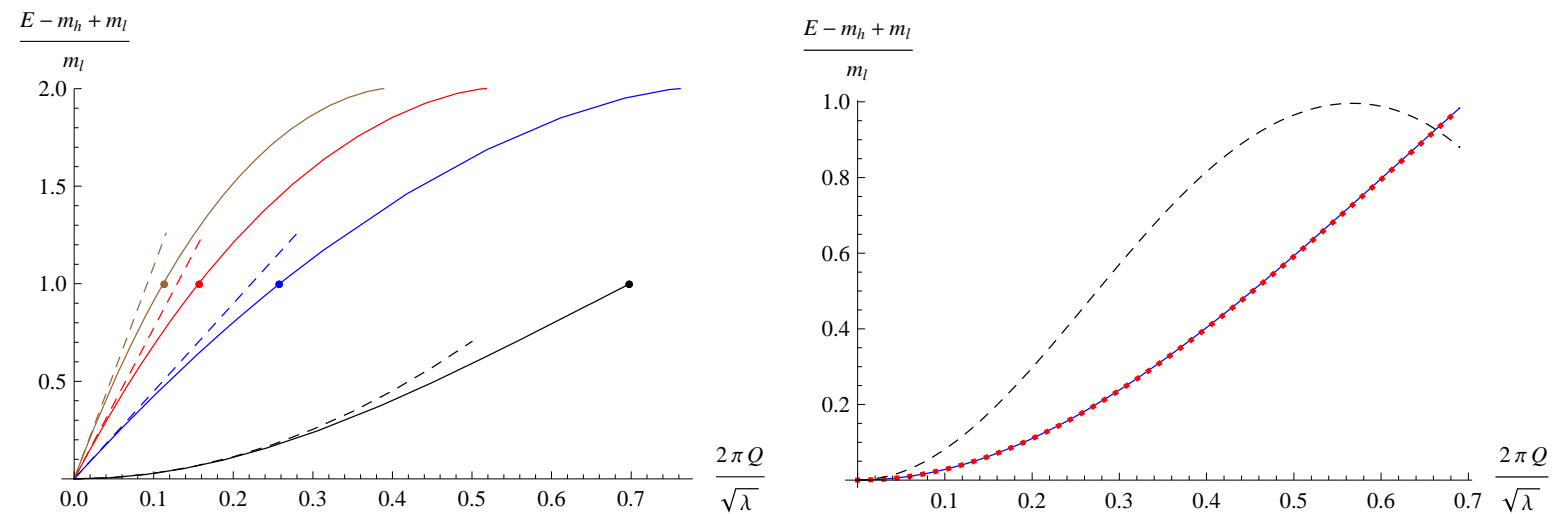

Figure 7: Left: $E$ versus $Q$ for the spinning heavy-light mesons. From right to left, the solid curves correspond to the $n=0,1,2,3$ branches, and the corresponding dashed curves to the analytic small $Q$ approximations of eqs. (5.24) and (5.37). The value of $y_{h}$ is set to 100 , and the dots on the curves again denote the critical solutions. Right: The effect of changing the heavy brane location from $y_{h}=100$ (solid blue curve) to $y_{h}=10$ (dotted red) in the $n=0$ case. The difference of the two curves is also shown as the dashed black line, magnified by a factor of 500 .

on their small $Q$ limits. Similar to the analysis of the strings spinning in real space, we can consider the approximate solution, valid for small $A$,

$$
\begin{aligned}
\delta \rho & =A \frac{1}{z}\left(\omega_{n} z_{l} \cos \left(\omega_{n}\left(z-z_{l}\right)\right)+\sin \left(\omega_{n}\left(z-z_{l}\right)\right)\right), \\
\theta & =\omega_{n} t,
\end{aligned}
$$

with $z \equiv 1 / y$ and the $\omega_{n}$ 's given by our $\rho$ fluctuation spectrum. This solution leads to the approximate small $Q$ relation

$$
E \approx m_{h}-m_{l}+m_{l} \omega_{n} z_{l} \frac{2 \pi Q}{\sqrt{\lambda}}
$$

which is shown as the dashed straight lines in figure 7 (left). Like the small $J$ result (5.11), this linear dependence of $E$ on $Q$ is characteristic of a particle in a Hooke's Law potential.

Decreasing $\Omega$ towards the critical angular velocities $\Omega_{\mathrm{nc}}, n \geq 1$, we observe that the charge $Q$ approaches a critical value $Q_{\text {nc }}$, varying according to $n$, while the energy $E$ approaches a universal constant $E_{c} \approx m_{h}$, independent of the branch in question. Both values, as well as the forms of the $E(Q)$ curves, are highly independent of the location of the heavy brane at sufficiently large values of $y_{h}$, and for $y_{h} \geq 10$, the first few values of $Q_{\mathrm{nc}}$ are $Q_{1 c}=0.258 \sqrt{\lambda} / 2 \pi, Q_{2 c}=0.156 \sqrt{\lambda} / 2 \pi$, and $Q_{3 c}=0.112 \sqrt{\lambda} / 2 \pi$. This $m_{h}$ independence can be understood by inspecting the form of the canonical momentum densities appearing in eqs. (5.19)-(5.20). The charge density $\pi_{\theta}^{0}$ behaves at large $y$ as $1 / y^{4}$. The energy density scales at leading order as $\sqrt{\lambda}$, giving rise to the ground state mass $m_{h}-m_{l}$ of the heavylight meson, but the first correction also behaves as $1 / y^{4}$. These $1 / y^{4}$ terms mean that the excitation energy as a function of the charge of the spinning string is highly insensitive to the form of the string profile at $y \gtrsim 10 y_{l}$. 
If we proceed to even smaller frequencies, $0<\Omega<\Omega_{\mathrm{nc}}$, we notice that these $n>0$ branches persist all the way down to zero. In the limit $\Omega \rightarrow 0$, the strings become marginally bound, like their real-space spinning counterparts, with an energy $E \approx m_{h}+m_{l}$. In contrast, the charges $Q$ for the terminal solutions are not universal. For the case $m_{h}=$ $100 m_{l}$, we find that the terminal values of $2 \pi Q / \sqrt{\lambda}$ are $0.762,0.518$, and 0.390 for the $n=1,2$, and 3 branches respectively. Like their real-space spinning counterparts, we suspect that these long strings are not stable for the exact same reasons.

Switching then to following the $n=0$ branch on the $(Q, E)$ plane, we observe that for a given value of the charge, these strings are always energetically favored in comparison with their $n \geq 1$ counterparts. In the limit $y_{h} \rightarrow \infty$, we find that the energy and charge of the critical solution on the $n=0$ branch very quickly approach

$$
\begin{aligned}
E_{0 c} & =m_{h}-6(1) \frac{m_{l}^{4}}{m_{h}^{3}}+\mathcal{O}\left(\frac{m_{l}^{5}}{m_{h}^{4}}\right), \\
\frac{2 \pi Q_{0 c}}{\sqrt{\lambda}} & =0.69868(1)-4.0(5) \frac{m_{l}^{3}}{m_{h}^{3}}+\mathcal{O}\left(\frac{m_{l}^{4}}{m_{h}^{4}}\right),
\end{aligned}
$$

where the coefficients of the first terms have been found by fitting a variety of trial functions to our numerical data and the errors have been estimated in a very conservative manner. The vanishing of the first few corrections in $1 / m_{h}$ is similar to the suppression of $1 / m_{h}$ corrections in the $\rho$ fluctuation analysis of section 4.3. The $n=0$ branch does not appear to admit long string solutions.

Small $\boldsymbol{\Omega}$ limit of the $\boldsymbol{n}=\mathbf{0}$ branch. To conclude our inspection of the string spinning in the $\theta$ direction, we will now take a closer look at the limit of infinitesimally small $\Omega$ in order to gain more understanding of the behavior of the $n=0$ solutions there. We note that this limit corresponds to approximating $y_{l} \gg \Omega$, and therefore implies that we may use the relation $u^{4}-\Omega^{2} \rho^{2} \approx u^{4}$ in the equation of motion for $\rho$. On the other hand, the observed fact that $\rho$ is nearly a constant in this case implies that

$$
\left(u^{2}-2 \rho^{2}+2 y_{5} \rho \rho^{\prime}\right)\left(1+\left(\rho^{\prime}\right)^{2}\right) \rho \approx\left(u^{2}-2 \rho^{2}\right) \rho,
$$

finally giving as the equation to solve

$$
u^{6} \rho^{\prime \prime}+\Omega^{2}\left(u^{2}-2 \rho^{2}\right) \rho=0 .
$$

In the last form, we note that we may write

$$
\rho(y)=\rho_{0}+\delta \rho(y)
$$

where $\rho_{0}$ is a constant and $\delta \rho(y)$ satisfies the Neumann boundary conditions at $y=y_{l}$ and $y=y_{h}$. We define $\rho_{0}$ by the constraint that $\delta \rho \rightarrow 0$, as $y \rightarrow y_{h}$. Using this parametrization and the fact that $y_{l} \gg \Omega$, we see that $\delta \rho$ satisfies the equation of motion

$$
\delta \rho^{\prime \prime}=-\Omega^{2} \frac{\left(y^{2}-\rho_{0}^{2}\right)}{\left(y^{2}+\rho_{0}^{2}\right)^{3}} \rho_{0} .
$$


If we enforce the boundary condition at $y=y_{h}$, this differential equation can then be integrated to yield

$$
\begin{aligned}
\delta \rho(y) / \Omega^{2}= & \frac{\left(y-y_{h}\right)\left(\rho_{0}^{4}\left(y-2 y_{h}\right)-\rho_{0}^{2} y y_{h}\left(3 y-y_{h}\right)-y^{2} y_{h}^{3}\right)}{4 \rho_{0}\left(y^{2}+\rho_{0}^{2}\right)\left(y_{h}^{2}+\rho_{0}^{2}\right)^{2}} \\
& +\frac{y}{4 \rho_{0}^{2}}\left(\arctan \left[\frac{y}{\rho_{0}}\right]-\arctan \left[\frac{y_{h}}{\rho_{0}}\right]\right),
\end{aligned}
$$

from where - demanding that the derivative of this expression vanish also at $y=y_{l}$ we finally obtain as the equation for $\rho_{0}$

$$
\frac{\left(y_{l}^{2}+3 \rho_{0}^{2}\right) y_{l} \rho_{0}}{\left(y_{l}^{2}+\rho_{0}^{2}\right)^{2}}-\frac{\left(y_{h}^{2}+3 \rho_{0}^{2}\right) y_{h} \rho_{0}}{\left(y_{h}^{2}+\rho_{0}^{2}\right)^{2}}=\arctan \left[\frac{y_{h}}{\rho_{0}}\right]-\arctan \left[\frac{y_{l}}{\rho_{0}}\right] .
$$

Solving this equation numerically produces two solutions, $\rho_{0}=0$ and $\rho_{0}=F\left(y_{h} / y_{l}\right) \times y_{l}$, of which we can throw out the former, as it is not consistent with our assumption of a small $\delta \rho$ and furthermore leads to a vanishing angular momentum. The latter result, on the other hand, is a slowly varying function of $y_{h} / y_{l}$ for large values of this ratio, approaching in the $y_{h} / y_{l} \rightarrow \infty$ limit the result $\rho_{0} \approx 1.82526 y_{l}$. In contrast, for $y_{h} \approx y_{l}, F\left(y_{h} / y_{l}\right) \approx 1$.

Properties of the small- $\Omega$ solution. To get some feeling for the physical properties of the above solutions obtained for small $\Omega \ll y_{l}$, we will next compute their energy $E$ and internal angular momentum $Q$ using eq. (5.19), where the canonical momentum and internal angular momentum densities read approximately

$$
\pi_{t}^{0} \approx-\frac{L^{2}}{2 \pi \alpha^{\prime}}\left(1+\frac{\rho_{0}^{2} \Omega^{2}}{2 u_{0}^{4}}\right) \quad \text { and } \quad \pi_{\theta}^{0} \approx \frac{L^{2}}{2 \pi \alpha^{\prime}} \frac{\rho_{0}^{2} \Omega}{u_{0}^{4}},
$$

with $u_{0}^{2} \equiv y^{2}+\rho_{0}^{2}$. Here, we have neglected higher order corrections in $\Omega$ and used the approximate solution (5.31). Performing the integrals, we obtain

$$
E \approx \frac{L^{2}}{2 \pi \alpha^{\prime}}\left(y_{h}-y_{l}+\frac{\Omega^{2}}{2 y_{l}} \Upsilon\right) \quad \text { and } \quad Q \approx \frac{L^{2}}{2 \pi \alpha^{\prime}} \frac{\Omega}{y_{l}} \Upsilon
$$

in which we have defined the dimensionless constant

$$
\Upsilon \equiv \rho_{0}^{2} y_{l} \int_{y_{l}}^{y_{h}} d y \frac{1}{\left(y^{2}+\rho_{0}^{2}\right)^{2}}=\rho_{0}^{2} y_{l}\left(\frac{y_{l}}{\left(\rho_{0}^{2}+y_{l}^{2}\right)^{2}}-\frac{y_{h}}{\left(\rho_{0}^{2}+y_{h}^{2}\right)^{2}}\right) .
$$

In deriving eq. (5.35), we have made use of eq. (5.32). Note that we have

$$
\lim _{y_{h} \rightarrow \infty} \Upsilon \approx 0.17757 \quad \text { while } \quad \lim _{y_{h} \rightarrow y_{l}} \Upsilon=\frac{y_{h}-y_{l}}{4 y_{l}} .
$$

We may now easily solve $\Omega$ in terms of $Q$ from eq. (5.34) above, which allows us to write $E$ in terms of $Q$

$$
E \approx m_{h}-m_{l}+\frac{m_{l}}{2 \Upsilon}\left(\frac{2 \pi Q}{\sqrt{\lambda}}\right)^{2}
$$

Thus we find again that the excitation spectrum does not depend on $m_{h}$ at leading order in the heavy quark mass limit. As we can see from figure 7 , this analytic approximation is quite good even for moderately large values of $Q$. The quadratic dependence of $E$ on $Q$ is characteristic of a particle in a square well, $\theta\left(r-r_{0}\right)$, type potential. 


\section{Summary and discussion}

Although different in many respects, the heavy-light mesons we have studied have a spectrum which shares certain properties of real-world heavy-light mesons. For example, consider the case where there are two heavy quarks $h$ and $h^{\prime}$ and two light quarks $l$ and $l^{\prime}$. We find for the ground state heavy-light mesons that

$$
M_{h l}-M_{h l^{\prime}}=m_{l^{\prime}}-m_{l}=M_{h^{\prime} l}-M_{h^{\prime} l^{\prime}}
$$

This kind of relation is similar to the real world relation (see for example ref. [1]) for mesons containing a charm or bottom quark,

$$
m_{B_{s}}-m_{B} \approx m_{D_{s}}-m_{D} \approx 100 \mathrm{MeV} .
$$

Of course, the sign of the above difference is wrong: While for us, given that $m_{l}>m_{l^{\prime}}$, we would find a negative difference, in the real world the difference is positive. This sign difference is, however, of little significance in this $\mathcal{N}=2 \mathrm{SYM}$ theory. In section 2, we noted that we could let the lighter D7 brane end along $w^{3}=c^{\prime}$ where $c^{\prime} \in \mathbb{C}$ and $\left|c^{\prime}\right|=1 / z_{l}$. This case still preserves $\mathcal{N}=2$ supersymmetry and allows us to tune the mass of the ground state heavy-light meson to be anything between $m_{h}-m_{l}$ and $m_{h}+m_{l}$. We did not study the excitation spectra of these more general heavy-light mesons in this paper, but it would be an interesting project for the future.

What we calculated was a portion of the heavy-light meson spectrum for hypermultiplets with masses with the same phase. In the dual language, both of our D7 branes sit at $y_{6}=0$ (or equivalently $\operatorname{Im} w^{3}=0$ ) and different values of $y_{5}$. One generic feature of this spectrum is the $m_{h}$ independence of the excitation energies in the heavy quark mass limit. For example, for low lying fluctuations in the $x$ and $y_{6}$ directions we found the energy spectrum

$$
E_{n}=m_{h}-m_{l}+m_{l} \frac{2 \pi^{2} n}{\sqrt{\lambda}}+\mathcal{O}\left(\frac{m_{l}^{2}}{m_{h}}\right) .
$$

For the $\rho$ fluctuations, we were not able to determine a spectrum analytically, but were nevertheless able to determine this $m_{h}$ independence numerically. The $x$ fluctuations should correspond to vector like mesons, while the $y_{6}$ and $\rho$ fluctuations should correspond to scalar like mesons.

We also studied spinning strings. For the strings spinning in real space, we found several branches, characterized by a radial excitation number $n$. For small angular momentum $J$, we were able to determine the analytic formula

$$
E=m_{h}-m_{l}+m_{l} \frac{2 \pi^{2} n J}{\sqrt{\lambda}}+\mathcal{O}\left(\frac{m_{l}^{2}}{m_{h}}\right)
$$

which displays this $m_{h}$ independence. Finally we studied strings spinning in an internal space, which corresponds to mesons with R-charge $Q$ from the field theory perspective. For small $Q$, we found the analytic formulae of eqs. (5.24) and (5.37) which again displays $m_{h}$ independence. 
Continuing the comparison with QCD, we can consider the mass difference between an excited and a ground state heavy-light meson in QCD. From the review [1], we learn that a typical QCD prediction of this heavy quark limit is that the difference in energy between excited and ground state heavy-light mesons should obey the relations

$$
m_{B_{2}^{*}}-m_{B} \approx m_{D_{2}^{*}}-m_{D} \approx 593 \mathrm{MeV}, \quad m_{B_{1}}-m_{B} \approx m_{D_{1}}-m_{D} \approx 557 \mathrm{MeV} .
$$

Unfortunately, there is no good data yet for $m_{B_{2}^{*}}$ and $m_{B_{1}}$. These differences are consistent with our result that the energy excitations scale with $m_{l}$, although in real world QCD, we expect to have $m_{l}$ replaced with $\Lambda_{\mathrm{QCD}}$.

The electromagnetic mass splittings of heavy-light mesons in QCD are typically tiny [22]. For example, $m_{D^{ \pm}}-m_{D^{0}} \approx 5 \mathrm{MeV}$ while $m_{B^{0}}-m_{B^{ \pm}} \approx 0.4 \mathrm{MeV}$. It is suggestive that in the large $\lambda$ limit, our approximate formula (5.37) for the $Q$ dependence of the masses is suppressed by an additional power of $Q / \sqrt{\lambda}$ compared with the linear scaling of eq. (6.4) on $J / \sqrt{\lambda}$. However, we have no good understanding of the relative sizes of the splittings for these $D$ and $B$ mesons.

One interesting phenomenon in QCD that we did not observe in our AdS/CFT model is hyperfine splitting. There are special pairs of mesons in QCD, which differ by the spin of the heavy quark and for which the mass difference is proportional to $\Lambda_{\mathrm{QCD}}^{2} / m_{h}$. In our fluctuation analysis, there are degeneracies in the spectra, which might provide a starting point to look for these hyperfine effects. For example, the lowest lying excitation in the $x$ direction is a vector meson with the same energy as the scalar meson corresponding to the lowest lying excitation in the $y_{6}$ direction. This degeneracy is likely a consequence of $\mathcal{N}=2$ supersymmetry, and we expect the fermionic fluctuations of the superstring will fill out this $\mathcal{N}=2$ massive supermultiplet. It is tempting to speculate that in a background with $\mathcal{N}=1$ or no supersymmetry, the energies of the vector and scalar mesons will develop a hyperfine splitting. ${ }^{10}$

Finally, we make some comments regarding two specific open questions related to our work.

Hybrid mesons. In phenomenological QCD literature, one finds discussion of hybrid mesons. In perturbative language, such an object would be a bound state of a quark, antiquark, and gluon [23], while at strong coupling, there exist models of a heavy quark and antiquark joined by a vibrating flux tube [24]. This second picture is similar to but also rather different from our model. Like us, the authors of ref. [24] begin by finding the modes of the vibrating flux tube joining the quarks. However, in their model, both quarks are heavy. Also, and perhaps more importantly, the quarks themselves have a mass large compared to the energy of the flux tube, whereas in ours, the mass of the meson is the mass of the flux tube. As a next step, the authors of ref. [24] use the vibrating flux tube to construct a phenomenological Cornell like potential through which the massive quarks interact. Despite these differences, one wonders if there exists a closer connection between our heavy-light mesons in $\mathcal{N}=2 \mathrm{SYM}$ and hybrid heavy-light mesons in QCD - if such things exist — rather than the "ordinary" heavy-light mesons of QCD.

\footnotetext{
${ }^{10}$ We would like to thank J. Erdmenger and D. Son for discussion on this point.
} 
W bosons. One may also consider Higgsing the $\mathcal{N}=4 \mathrm{SU}(N)$ SYM theory down to $\mathrm{SU}(N-2) \times \mathrm{U}(1)^{2}$. In the dual gravitational picture, this Higgsing corresponds to pulling two D3 branes off of the stack of $N$ D3 branes, whose low energy description this SYM theory is. As long as we keep the D3 branes parallel in this $A d S_{5} \times S^{5}$ geometry, they do not experience a potential and we can imagine placing them at nonzero values of $y$, just as we did for the D7 branes. There is then a semi-classical string that stretches between the two D3 branes, whose fluctuations we may study and which has a dual field theory interpretation as a $\mathrm{W}$ boson. ${ }^{11}$

We mention this D3 brane and string construction because we can at this point in our analysis treat it very easily. The treatment of the string spinning in real space and corresponding to a heavy-light meson is identical for the $\mathrm{W}$ bosons. Also, the $x$ fluctuations of such a string are identical to the $x$ fluctuations for the heavy-light meson. Finally, the $y_{6}$ fluctuations are identical, except that there are now four additional $y_{6}$-like directions perpendicular to the D3 brane string configuration. Whereas for the heavy-light meson, the $x$ and $y_{6}$ fluctuations gave us four towers of identical modes, and the $\rho$ fluctuations gave us another four towers, for the $\mathrm{W}$ boson, the $x$ and $y_{6}$ fluctuations give us eight towers of identical modes. We believe this regrouping of one pair of four identical towers into eight identical towers is related to the doubling in the amount of supersymmetry. The $\mathcal{N}=2$ SYM relevant for the heavy-light mesons has eight supercharges, whereas the $\mathcal{N}=4 \mathrm{SYM}$, after the Higgsing which breaks conformal invariance, should have 16.

\section{Acknowledgments}

We would like to thank J. Erdmenger, N. Evans, Ph. de Forcrand, K. Kajantie, D. Kaplan, I. Klebanov, A. Kurkela, D. Mateos, A. Rebhan, D. Rodriguez-Gomez, A. Scardicchio, and D. Son for valuable discussions. We would also like to thank A. Paredes and P. Talavera for bringing their work [14] to our attention. C.P.H. would like to thank the KITP, where part of this work was done, for hospitality. C.P.H. was supported in part by the National Science Foundation under Grants No. PHY-0243680 and PHY05-51164, S.A.S. by the Austrian Science Foundation, FWF, project No. P19958, and A.V. in part by the Austrian Science Foundation, FWF, project No. M1006.

\section{References}

[1] M. Neubert, Heavy quark symmetry, Phys. Rept. 245 (1994) 259 hep-ph/9306320.

[2] A. Karch and E. Katz, Adding flavor to AdS/CFT, JHEP 06 (2002) 043 hep-th/0205236.

[3] J.M. Maldacena, The large- $N$ limit of superconformal field theories and supergravity, Adv. Theor. Math. Phys. 2 (1998) 231 [Int. J. Theor. Phys. 38 (1999) 1113 hep-th/9711200].

[4] E. Witten, Anti-de Sitter space and holography, Adv. Theor. Math. Phys. 2 (1998) 253 hep-th/9802150.

[5] S.S. Gubser, I.R. Klebanov and A.M. Polyakov, Gauge theory correlators from non-critical string theory, Phys. Lett. B 428 (1998) 105 hep-th/9802109.

\footnotetext{
${ }^{11}$ We would like to thank I. Klebanov for suggesting we think about this extension of our results.
} 
[6] I.R. Klebanov and M.J. Strassler, Supergravity and a confining gauge theory: duality cascades and $\chi$ SB-resolution of naked singularities, JHEP 08 (2000) 052 hep-th/0007191.

[7] E. Shuryak, Why does the quark gluon plasma at RHIC behave as a nearly ideal fluid?, Prog. Part. Nucl. Phys. 53 (2004) 273 hep-ph/0312227; What RHIC experiments and theory tell us about properties of quark-gluon plasma?, Nucl. Phys. A 750 (2005) 64 hep-ph/0405066.

[8] S.S. Gubser, I.R. Klebanov and A.W. Peet, Entropy and temperature of black 3-branes, Phys. Rev. D 54 (1996) 3915 hep-th/9602135.

[9] P. Romatschke and U. Romatschke, Viscosity information from relativistic nuclear collisions: how perfect is the fluid observed at RHIC?, Phys. Rev. Lett. 99 (2007) 172301 arXiv:0706.1522.

[10] G. Policastro, D.T. Son and A.O. Starinets, The shear viscosity of strongly coupled $N=4$ supersymmetric Yang-Mills plasma, Phys. Rev. Lett. 87 (2001) 081601 hhep-th/0104066.

[11] A. Karch, E. Katz and N. Weiner, Hadron masses and screening from AdS Wilson loops, Phys. Rev. Lett. 90 (2003) 091601 hep-th/0211107.

[12] M. Kruczenski, D. Mateos, R.C. Myers and D.J. Winters, Meson spectroscopy in AdS/CFT with flavour, JHEP 07 (2003) 049 hep-th/0304032.

[13] J. Erdmenger, N. Evans, I. Kirsch and E. Threlfall, Mesons in gauge/gravity duals - a review, Eur. Phys. J. A35 (2008) 81 arXiv:0711.4467.

[14] A. Paredes and P. Talavera, Multiflavour excited mesons from the fifth dimension, Nucl. Phys. B 713 (2005) 438 hep-th/0412260.

[15] J. Erdmenger, N. Evans and J. Grosse, Heavy-light mesons from the AdS/CFT correspondence, JHEP 01 (2007) 098 hep-th/0605241.

[16] J. Erdmenger, K. Ghoroku and I. Kirsch, Holographic heavy-light mesons from non-Abelian $D B I$, JHEP 09 (2007) 111 arXiv: 0706.3978.

[17] C.P. Herzog, A. Karch, P. Kovtun, C. Kozcaz and L.G. Yaffe, Energy loss of a heavy quark moving through $N=4$ supersymmetric Yang-Mills plasma, JHEP 07 (2006) 013 hep-th/0605158.

[18] A.L. Cotrone, L. Martucci and W. Troost, String splitting and strong coupling meson decay, Phys. Rev. Lett. 96 (2006) 141601 hep-th/0511045.

[19] K. Peeters, J. Sonnenschein and M. Zamaklar, Holographic decays of large-spin mesons, JHEP 02 (2006) 009 hep-th/0511044.

[20] P.M. Chesler and A. Vuorinen, Heavy flavor diffusion in weakly coupled $N=4$ super Yang-Mills theory, JHEP 11 (2006) 037 hep-ph/0607148.

[21] J. Gomis, F. Marchesano and D. Mateos, An open string landscape, JHEP 11 (2005) 021 hep-th/0506179.

[22] Particle Data Group collaboration, W.-M. Yao et al., Review of particle physics, J. Phys. G 33 (2006) 1; http://pdg.lbl.gov/.

[23] R.L. Jaffe and K. Johnson, Unconventional states of confined quarks and gluons, Phys. Lett. B 60 (1976) 201.

[24] N. Isgur and J.E. Paton, A flux tube model for hadrons in QCD, Phys. Rev. D 31 (1985) 2910 . 